\title{
Rate of Convergence Estimates for Nonselfadjoint Eigenvalue Approximations*
}

\author{
By J. H. Bramble and J. E. Osborn
}

\begin{abstract}
In this paper, a general approximation theory for the eigenvalues and corresponding subspaces of generalized eigenfunctions of a certain class of compact operators is developed. This theory is then used to obtain rate of convergence estimates for the errors which arise when the eigenvalues of nonselfadjoint elliptic partial differential operators are approximated by Rayleigh-Ritz-Galerkin type methods using finite-dimensional spaces of trial functions, e.g. spline functions. The approximation methods include several in which the functions in the space of trial functions are not required to satisfy any boundary conditions.
\end{abstract}

1. Introduction. In this paper, we develop a general approximation theory for the eigenvalues and corresponding subspaces of generalized eigenvectors for a certain class of compact operators. This theory is then applied to several examples. The principal examples consist of certain Rayleigh-Ritz-Galerkin type methods for the approximation of the eigenvalues of second-order nonselfadjoint elliptic differential operators. In each case we give estimates for the rate of convergence. By means of our general theorems on compact operators, the problem of estimating the rate of convergence of the eigenvalues is reduced to obtaining estimates for the rate of convergence in the approximate solution of the corresponding boundary value problem. We make strong use of not only $\mathfrak{L}_{2}$ estimates but also estimates in Sobolev spaces of negative order.

Although our theorems could be applied to higher-order elliptic equations, ordinary differential equations and integral operators, we have chosen to center our attention on the general second-order elliptic case and to obtain specific results for a variety of projection methods.

There is an extensive literature dealing with the approximation of eigenvalues of selfadjoint operators. For the case of a selfadjoint operator which is bounded below, upper bounds for the eigenvalues are provided by the Rayleigh-Ritz method. The method of intermediate problems introduced by Weinstein [47], [48] provides lower bounds. This method was extended by Weinstein [49], [50], [51], [52], Aronszajn and Weinstein [3], Aronszajn [1], [2], Weinberger [45], [46], Bazley [7], Bazley and Fox [8], [9], and Stenger [41]. Another method has been developed by Fichera [21], [22] for finding lower bounds for eigenvalues connected with selfadjoint boundary value problems for elliptic differential operators in a bounded domain.

Also, in the selfadjoint case, Birkhoff, de Boor, Swartz, and Wendroff [11] prove an inequality from which lower bounds can be derived for the eigenvalues in terms

Received June 8, 1972.

AMS (MOS) subject classifications (1970). Primary 35P15, 65N15, 65N25, 65N30.

* Sponsored by the United States Army under Contract No. DA-31-124-ARO-D-462. 
of the Rayleigh-Ritz eigenvalues corresponding to a given finite-dimensional subspace and the approximability properties of the subspace. A specific application of this was carried out for second-order ordinary differential operators using subspaces of cubic splines. This idea has been generalized so as to apply to selfadjoint elliptic operators and rather general finite-dimensional subspaces; cf. Ciarlet, Schultz, and Varga [19], Schultz [39], [40]. Pierce and Varga [33], [34] give $\mathfrak{L}_{2}$ eigenvector estimates which are optimal in the sense of corresponding to the approximability properties of the underlying subspaces.

Perhaps the work closest to that of this paper is the work of Vainnikko [42], [43], [44]. In [44], he proves a general theorem on convergence of eigenvalues and generalized eigenvectors. Our Theorems 3 and 4 of Section 3 are analogous to his. He does not obtain results of the type given in Theorems 1 and 2 of Section 3 (our main theorems) and it is not clear how to put several of our applications into his setting. His strongest results rest on the fact that an operator $T$ is approximated by an operator $P_{h} T$ where $P_{h}$ is a certain projection whose adjoint can be calculated. This task, which must be carried out with each application, does not appear to be simple. Our approach, while avoiding this difficulty, yields stronger results.

Recently, Osborn [30], [31], [32] has derived error estimates for Galerkin approximations of the eigenvalues of nonselfadjoint ordinary differential operators which are lower order perturbations of selfadjoint operators. The eigenfunctions of the related selfadjoint operator are used as trial functions and the error estimates depend in a simple way on the data of the problem.

We mention finally that Marek [27] gives error estimates for the approximation of the Perron root of a class of nonselfadjoint operators which are of positive type provided the approximate operator is also of positive type.

Error estimates for the approximate calculation of eigenvalues of selfadjoint operators have always depended on a variational characterization of the eigenvalues. In the present work, no use is made of such variational principles; instead, we use certain estimates for the related resolvent operator. Special features of this work are:

1. Approximations for the generalized eigenfunctions are obtained first and then used to obtain the eigenvalue estimates.

2. In the case of a multiple eigenvalue (in general having different algebraic and geometric multiplicities), a weighted average of approximate eigenvalues is shown to be the "right" choice as an approximation.

3. The results apply to general nonselfadjoint elliptic operators.

4. Various methods for defining the approximate eigenvalues are treated, including methods in which the functions in the subspaces of trial functions are not required to satisfy any boundary conditions.

5. The estimates are optimal in the sense that they give the best estimate that could be expected on the basis of the approximability properties of the underlying subspaces.

An outline of the paper is as follows: In Sections 2 and 3, we derive results on the approximation of eigenvalues and generalized eigenfunctions of a class of compact operators. Section 4 deals with the general class of differential eigenvalue problems to be considered in the paper. In Section 5 we discuss the families of finite-dimensional subspaces used in constructing the approximations. Sections 6-11 contain various smethods for constructing the approximate eigenvalues: The ordinary Galerkin 
method, the least squares method of Bramble and Schatz [15], [16], methods of Nitsche [28], [29], and a recent method of Babuška [6]. In each case the eigenvalue results are shown to follow easily from results on the approximation of the solution of the corresponding inhomogeneous problem.

2. General Notation. Let $\Omega$ be a bounded domain in Euclidean $N$-dimensional space $R^{N}$ with boundary $\partial \Omega$ which we will assume (for convenience) to be of class $C^{\infty}$. Let $C^{\infty}(\bar{\Omega})$ be the class of infinitely differentiable complex valued functions defined on $\bar{\Omega}$, the closure of $\Omega$. For functions in $C^{\infty}(\bar{\Omega})$, we define the $\mathfrak{L}_{2}$-inner product by

$$
(\varphi, \psi)=\int_{\Omega} \varphi \bar{\psi} d x
$$

and the corresponding norm by

$$
\|\varphi\|_{0}=\left(\int_{\Omega}|\varphi|^{2} d x\right)^{1 / 2}
$$

Let $\alpha=\left(\alpha_{1}, \cdots, \alpha_{N}\right)$ be a multi-index (i.e., each $\alpha_{i}, i=1, \cdots, N$, is a nonnegative integer),

$$
D^{\alpha}=\partial^{\alpha_{1}} / \partial x_{1}^{\alpha_{1}} \cdots \partial^{\alpha_{N}} / \partial x_{N}^{\alpha_{N}}
$$

and $|\alpha|=\sum_{j=1}^{N} \alpha_{i}$. We define for $\varphi \in C^{\infty}(\bar{\Omega})$ and for $j$ a nonnegative integer the norm

$$
\|\varphi\|_{i}=\left(\sum_{|\alpha| \leqq i}\left\|D^{\alpha} \varphi\right\|_{0}^{2}\right)^{1 / 2}
$$

The completion of $C^{\infty}(\bar{\Omega})$ with respect to $\|\cdot\|_{i}$ will be denoted by $H^{i}(\Omega)$. This is the Sobolev space of order $j$ and is a Hilbert space. For any positive real number $s$, we define $H^{s}(\Omega)$ by interpolation between successive integers following Definition 2.1 of [26]. The spaces $H^{s}(\Omega)$ for $s<0$ are defined as follows:

For $\varphi \in C^{\infty}(\bar{\Omega})$ and $s<0$, set

$$
\|\varphi\|_{s}=\sup _{\psi \in C^{\infty}(\bar{\Omega})}|(\varphi, \psi)| /\|\psi\|_{-s} .
$$

Then $H^{s}(\Omega)$, for $s<0$, is defined as the completion of $C^{\infty}(\bar{\Omega})$ with respect to the norm $\|\cdot\|_{s}$ (cf. [10]). Now $H^{s}(\Omega)$ is a Hilbert space for any real $s$. Note that $H^{0}(\Omega)=$ $\mathscr{L}_{2}(\Omega)$ and that $H^{s_{2}}(\Omega) \subset H^{s_{1}}(\Omega)$ if $s_{1} \leqq s_{2}$. We state the following well-known result.

LEMMA 2.1 (Rellich). Let $s_{1}$ and $s_{2}$ be real numbers with $s_{1}<s_{2}$, and let $I_{s_{1}, s_{2}}$ be the injection operator mapping $H^{s_{2}}(\Omega)$ into $H^{s_{1}}(\Omega)$. Then $I_{s_{1}, s_{2}}$ is compact.

For any $A: H^{s}(\Omega) \rightarrow H^{s}(\Omega)$, we define, for $s_{1} \leqq s \leqq s_{2}$,

$$
\|A\|_{s_{1}, s_{2}}=\sup _{\varphi \in H^{s_{2}(\Omega)}}\|A \varphi\|_{s_{1}} /\|\varphi\|_{s_{2}} \text {. }
$$

Note that, for each given $s_{1}, s_{2}$ and $s$, there is a constant $C$ such that

$$
\|A\|_{s_{1, s}} \leqq C\|A\|_{s, s} .
$$


The restriction of $A$ to a subset $X$ of $H^{s}(\Omega)$ will be denoted by $\left.A\right|_{X}$. Let $\sigma(A)$ denote the spectrum of $A$ and $\rho(A)$ the resolvent set. For any complex number $z \in \rho(A)$, the resolvent operator $R_{z}(A)$ is defined by

$$
R_{z}(A)=(z-A)^{-1} .
$$

If $A$ is compact, then $\sigma(A)$ consists of a denumerable set of nonzero complex numbers and zero. Each nonzero point $\mu$ of $\sigma(A)$ is an eigenvalue of $A$; i.e., there exists a nonzero element $u$ of $H^{s}(\Omega)$ such that $A u=\mu u$. Zero may or may not be an eigenvalue.

Let $\mu$ be a nonzero eigenvalue of $A$. The least integer $\alpha$ such that $\mathscr{N}\left((A-\mu)^{\alpha}\right)=$ $\mathfrak{K}\left((A-\mu)^{\alpha+1}\right)$, where $\mathfrak{N}$ denotes the null space, is called the ascent of $A-\mu$. If $A$ is compact $\alpha$ is finite. The integer $m=\operatorname{dim} \mathfrak{N}\left((A-\mu)^{\alpha}\right)$ is called the algebraic multiplicity of $\mu$ and is likewise finite. The subspace $\mathfrak{T}\left((A-\mu)^{\alpha}\right)$ is called the space of generalized eigenfunctions corresponding to the eigenvalue $\mu$. The geometric multiplicity is equal to $\operatorname{dim} \Re(A-\mu)$ and is always less than or equal to $m$. In the case of a selfadjoint operator, the geometric and algebraic multiplicities of a given eigenvalue $\mu$ are equal.

We next state a general result on the approximation of eigenvalues of compact operators.

Let $K: \mathfrak{L}_{2}(\Omega) \rightarrow \mathfrak{L}_{2}(\Omega)$ be compact. Suppose that $\left\{K_{h}\right\}_{0<h \leqq 1}$ is a family of compact operators $K_{h}: \mathfrak{L}_{2}(\Omega) \rightarrow \mathfrak{L}_{2}(\Omega)$ such that

$$
\lim _{h \rightarrow 0}\left\|K-K_{h}\right\|_{0,0}=0 .
$$

Let $\mu_{1}, \mu_{2}, \cdots$ be the nonzero eigenvalues of $K$ ordered by decreasing magnitude taking account of algebraic multiplicities. Then for each $h>0$ there is an ordering of the eigenvalues of $K_{h}, \mu_{1}(h), \mu_{2}(h), \cdots$, such that, for each integer $j, \lim _{h \rightarrow 0} \mu_{j}(h)=$ $\mu_{i}$ (cf. [20]).

For the purpose of this paper, we shall restate this convergence property in the following convenient form.

LEMMA 2.2. Let $\mu$ be a nonzero eigenvalue of $K$ with algebraic multiplicity $m$ and let $\Gamma$ be a circle centered at $\mu$ which lies in $\rho(K)$ and contains no other points of $\sigma(K)$. Then, there is an $h_{0}$ such that, for $0<h \leqq h_{0}$, there are exactly $m$ eigenvalues (counting algebraic multiplicities) of $K_{h}$ lying inside $\Gamma$ and all points of $\sigma\left(K_{h}\right)$ are bounded away from $\Gamma$.

The next lemma relates the projection onto the generalized null space corresponding to $\mu$ to the projection associated with the part of the spectrum of $K_{h}$ inside $\Gamma$ (cf. [25]).

LEMMA 2.3. With $\Gamma$ and $h_{0}$ as in Lemma 2.2, if $0<h \leqq h_{0}$, the operators

$$
E(\mu)=\frac{1}{2 \pi i} \int_{\Gamma} R_{z}(K) d z \quad \text { and } \quad E_{h}(\mu)=\frac{1}{2 \pi i} \int_{\Gamma} R_{z}\left(K_{h}\right) d z
$$

are projections (in general nonorthogonal). The range of $E(\mu), R(E(\mu))$, is the space of generalized eigenfunctions of $K$ corresponding to $\mu$ and $R\left(E_{h}(\mu)\right)$ is the direct sum of the spaces of generalized eigenfunctions of $K_{h}$ associated with the eigenvalues of $K_{h}$ inside of $\Gamma$. Finally, 


$$
\lim _{h \rightarrow 0}\left\|E(\mu)-E_{h}(\mu)\right\|_{0,0}=0
$$

and $\operatorname{dim} R\left(E_{h}(\mu)\right)=\operatorname{dim} R(E(\mu))=m$.

3. Convergence Estimates. In this section we will consider a particular type of compact operator $T$ and a family of compact operators $\left\{T_{h}\right\}_{0<h \leqq 1}$ which approximates it. The first theorem relates a certain invariant subspace of $T_{h}$ to generalized eigenfunctions of $T$. These estimates are then used to deduce estimates for the rate of convergence of the eigenvalues of $T_{h}$ to the eigenvalues of $T$.

More precisely let $s_{0}$ be a fixed nonnegative real number. Let $T$ be an operator from $H^{-s_{0}}(\Omega)$ to $H^{-s_{0}}(\Omega)$. We suppose further that for some fixed $\epsilon>0$ and all $s \geqq-s_{0}$,

$$
T: H^{*}(\Omega) \rightarrow H^{s+\epsilon}(\Omega)
$$

as a bounded mapping.

From (3.1) we see that $T$ can be considered as an operator from $H^{s}(\Omega)$ to $H^{s}(\Omega)$ for $s \geqq-s_{0}$ and, from Lemma 2.1 we see that $T$ will be compact as an operator on $H^{s}(\Omega)$. In the sequel the space $H^{s}(\Omega)$ on which we are considering $T$ will be clear from the context.

Lemma 3.1. For $s \geqq-s_{0}$, the spectrum of $T$ on $H^{s}(\Omega)$ is the same as the spectrum of $T$ on $\mathfrak{L}_{2}(\Omega)$.

Proof. Clearly, the spectrum of $T$ on $H^{-s}{ }^{\circ}(\Omega)$ contains the spectrum of $T$ on $H^{s}(\Omega)$. Suppose $\mu \neq 0$ is in the spectrum of $T$ on $H^{-s^{\circ}}(\Omega)$. Then, there is a nonzero $u \in H^{-s}{ }^{\circ}(\Omega)$ such that $T u=\mu u$. By virtue of (3.1), $u \in H^{t}(\Omega)$ for all $t \geqq-s_{0}$. Hence, $u \in H^{s}(\Omega)$ and it follows that $\mu$ is in the spectrum of $T$ on $H^{s}(\Omega)$.

Note that the space of generalized eigenfunctions corresponding to a nonzero eigenvalue is also independent of $s$. This follows easily from (3.1).

Now let $\left\{T_{h}\right\}_{1<h \leqq 1}$ be a one-parameter family of compact operators from $L_{2}(r)$ to $L_{2}(r)$ such that

$$
\lim _{h \rightarrow 0)}\left\|T-T_{h}\right\|_{1,0}=0
$$

Let $\mu$ be a nonzero eigenvalue of $T$ with algebraic multiplicity $m$. Let $\Gamma$ be a circle centered at $\mu$ which lies in $\rho(T)$ and contains no other points of $\sigma(T)$. From Lemma 2.2 applied to $T$ and $\left\{T_{h}\right\}_{0<h \leq 1}$ we see that there is an $h_{0}$ such that, for $0<h$ $\leqq h_{0}$ there are exactly $m$ eigenvalues (counting algebraic multiplicities) of $T_{h}$ inside of $\Gamma$. Throughout this section, $\mu, \Gamma$, and $h_{0}$, will be considered fixed and the $m$ eigenvalues of $T_{h}$ which lie inside $\Gamma$ are denoted by $\mu_{1}(h), \cdots, \mu_{m}(h)$.

Now, from Lemma 2.3, with $K=\left.T\right|_{\mathfrak{L}_{2}(\Omega)}, K_{h}=T_{h}$ and $h \leqq h_{0}$, the operators

$$
E(\mu)=\frac{1}{2 \pi i} \int_{\Gamma} R_{z}\left(\left.T\right|_{\mathcal{L}^{\prime}:(\Omega)}\right) d z \quad \text { and } \quad E_{h}(\mu)=\frac{1}{2 \pi i} \int_{\Gamma^{\prime}} R_{z}\left(T_{h}\right) d z
$$

are projections onto the space of generalized eigenfunctions of $T$ corresponding to $\mu$ and the direct sum of the spaces of generalized eigenfunctions of $T_{h}$ corresponding to $\mu_{1}(h), \cdots, \mu_{m}(h)$, respectively, and $\operatorname{dim} R\left(E_{h}(\mu)\right)=\operatorname{dim} R(E(\mu))=m$.

The following lemma is central to our development.

LEMMA 3.2. Let $s_{1}$ be a given nonnegative real number. Then there are constants $C$ and $h_{1}$ such that for any $u \in H^{*}(\Omega), 0<h \leqq h_{1}$ and $0 \leqq s \leqq s_{0}$, 


$$
\begin{aligned}
\| E(\mu) u- & E_{h}(\mu) u \|_{-s} \\
& \leqq C\left\{\left\|T-T_{h}\right\|_{-s, s_{1}}+\left\|T-T_{h}\right\|_{-s, 0}\left\|T-T_{h}\right\|_{0, s_{1}}\right\} \cdot\|u\|_{s_{1}} .
\end{aligned}
$$

Proof. By (2.1), for $0 \leqq s \leqq s_{0}$,

$$
\begin{aligned}
\left\|E(\mu) u-E_{h}(\mu) u\right\|_{-s} & =\sup _{\varphi \in C^{\infty}(\bar{\Omega})}\left|\left(\left[E(\mu)-E_{h}(\mu)\right] u, \varphi\right)\right| /\|\varphi\|_{s} \\
& =\frac{1}{2 \pi} \sup _{\varphi \in C^{\infty}(\bar{\Omega})}\left|\int_{\Gamma} \frac{\left(\left[R_{z}(T)-R_{z}\left(T_{h}\right)\right] u, \varphi\right)}{\|\varphi\|_{s}} d z\right| .
\end{aligned}
$$

Now let $z \in \Gamma$ be arbitrary. We have for any $\varphi \in H^{s}(\Omega)$,

$$
\left|\left(\left[R_{z}(T)-R_{z}\left(T_{h}\right)\right] u, \varphi\right)\right| /\|\varphi\|_{s} \leqq\left\|\left[R_{z}(T)-R_{z}\left(T_{h}\right)\right] u\right\|_{-s} .
$$

Consider now

$$
\begin{aligned}
{\left[R_{z}(T)-R_{z}\left(T_{h}\right)\right] u=} & R_{z}(T)\left(T-T_{h}\right) R_{z}(T) u \\
& -R_{z}(T)\left(T-T_{h}\right) R_{z}\left(T_{h}\right)\left(T-T_{h}\right) R_{z}(T) u .
\end{aligned}
$$

It follows easily from (3.6) that

$$
\begin{aligned}
\|\left[R_{z}(T)-\right. & \left.R_{z}\left(T_{h}\right)\right] u \|_{-s} \\
\leqq & \left.\left\|R_{z}(T)\right\|\right|_{-s,-s}\left\|T-T_{h}\right\|_{-s_{,} s_{1}}\left\|R_{z}(T)\right\|_{s_{1}, s_{1}}\|u\|_{s_{1}} \\
& +\left\|R_{z}(T)\right\|_{-s_{,-s}}\left\|T-T_{h}\right\|_{-s, 0}\left\|R_{z}\left(T_{h}\right)\right\|_{0,0} \\
& \cdot\left\|T-T_{h}\right\|_{0_{,}, s_{1}}\left\|R_{z}(T)\right\|\left\|_{s_{1}, s_{1}}\right\| u \|_{s_{1}} .
\end{aligned}
$$

Now by Lemma 3.1, $z$ is in the resolvent set of $T$ on $H^{t}(\Omega)$ for $-s_{0} \leqq t$. Since the resolvent of $T$ on $H^{t}(\Omega)$ is continuous as a function of $z$ and $\Gamma$ is compact, we have for some constant $C$ that

$$
\left\|R_{z}(T)\right\|_{t, t} \leqq C
$$

for all $z \in \Gamma$ and $-s_{0} \leqq t \leqq s_{1}$.

Furthermore,

$$
R_{z}\left(T_{h}\right)=\left(I+R_{z}(T)\left(T-T_{h}\right)\right)^{-1} R_{z}(T)
$$

provided $\left\|R_{z}(T)\left(T-T_{h}\right)\right\|_{0,0}<1$. By (3.3) and (3.8), $h_{1}$ may be chosen so that this condition is fulfilled uniformly for $0<h \leqq h_{1}$ and $z \in \Gamma$. Thus there is a constant $C$ such that for $0<h \leqq h_{1}$ and all $z \in \Gamma$,

$$
\left\|R_{z}\left(T_{h}\right)\right\|_{0,0} \leqq C .
$$

Using (3.9), (3.8), and (3.7), we have

$$
\begin{aligned}
\|\left[R_{z}(T)-\right. & \left.R_{z}\left(T_{h}\right)\right] u \|_{-s} \\
& \leqq C\left\{\left\|T-T_{h}\right\|_{-s, s_{1}}+\left\|T-T_{h}\right\|_{-s, 0}\left\|T-T_{h}\right\|_{0, s_{1}}\right\}\|u\|_{s_{1}} .
\end{aligned}
$$

Inequality (3.4) now follows from (3.10), (3.5) and (3.4).

The next lemma is an easy consequence of Lemma 3.2. In what follows, the term orthonormal will always refer to $\mathfrak{L}_{2}(\Omega)$. 
LEMMA 3.3. Let $\left\{u_{i}\right\}_{j=1}^{m}$ be an orthonormal basis for $R(E(\mu))$ and $s_{1} \geqq 0$. There exist constants $C_{1}$ and $h_{2}$ and a basis $\left\{\tilde{w}_{i}\right\}_{i=1}^{m}$ for $R\left(E_{h}(\mu)\right)$ such that for $0 \leqq s \leqq s_{0}$ and $0<h \leqq h_{2}$,

(3.11) $\max _{1 \leqq i \leqq m}\left\|u_{i}-\tilde{w}_{i}\right\|_{-s} \leqq C_{1}\left\{\left\|T-T_{h}\right\|_{-s_{, s_{1}}}+\left\|T-T_{h}\right\|_{-s, 0}\left\|T-T_{h}\right\|_{0, s_{1}}\right\}$.

Proof. Since $u_{i} \in R(E(\mu))$ and $E(\mu)$ is a projection, we have that $u_{i}=E(\mu) u_{i}$. Since $u_{j} \in H^{s_{1}}(\Omega)$ we may apply Lemma 3.2 with $u=u_{i}, j=1, \cdots, m$. Hence, choosing $C_{1}=C \max _{i}\left\|u_{j}\right\|_{s_{1}}$ with $C$ the constant from Lemma 3.2, (3.11) follows with $\tilde{w}_{i}=E_{h}(\mu) u_{j}$. Now by (2.2),

$$
\left.\begin{array}{l}
\left\|T-T_{h}\right\|_{-s, s_{1}} \\
\left\|T-T_{h}\right\|_{-s, 0} \\
\left\|T-T_{h}\right\|_{0, s_{1}}
\end{array}\right\} \leqq C^{\prime}\left\|T-T_{h}\right\|_{0,0}
$$

and hence, because of (3.2) there is a constant $h_{2}$ such that for $0<h \leqq h_{2}$,

$$
\sum_{j=1}^{m}\left\|u_{i}-\tilde{w}_{i}\right\|_{0}^{2}<1
$$

But from this it follows that the $\tilde{w}_{i}$ 's are linearly independent and hence a basis for the $m$-dimensional space $R\left(E_{h}(\mu)\right)$.

The first of our principal aims of this section is to obtain an estimate like (3.11) but for an orthonormal basis for $R\left(E_{h}(\mu)\right)$. To this end we prove the following general lemma. Because of our application we phrase this lemma in $\mathfrak{L}_{2}(\Omega)$ even though it is true with $\mathfrak{L}_{2}(\Omega)$ replaced by any inner product space.

LEMMA 3.4. Let $m$ be a positive integer. There is a constant $C_{m}$ such that, for $f_{1}, \cdots, f_{m}$ any linearly independent set in $\mathfrak{L}_{2}(\Omega)$ and $g_{1}, \cdots, g_{m}$ the corresponding Gram-Schmidt orthonormalization, we have

$$
\max _{1 \leqq k \leqq m}|| f_{k}-g_{k} \|_{0} \leqq C_{m} \max _{1 \leqq j, k \leqq m}\left|\left(f_{j}, f_{k}\right)-\delta_{j k}\right|,
$$

where $\delta_{j k}$ is the Kronecker delta.

Proof. We proceed by induction on $m$. Let $M=\max _{1 \leqq i, k \leqq m}\left|\left(f_{i}, f_{k}\right)-\delta_{i k}\right|$. Observe that

$$
||\left|f_{m}\left\|_{0}-1|\leqq||| f_{m}\right\|_{0}^{2}-1\right| \leqq M
$$

In particular, for $m=1$, we have $\left\|f_{1}-g_{1}\right\|_{0}=\left|\left\|f_{1}\right\|_{0}-1\right| \leqq M$. Now assume the result holds for $m-1$ with $m \geqq 2$ and a constant $C_{m-1}$. Set

$$
\tilde{g}_{m}=f_{m}-\sum_{i=1}^{m-1}\left(f_{m}, g_{i}\right) g_{i}
$$

and consider first the case $M \leqq 1$. Since

$$
\left|\left(f_{m}, g_{i}\right)\right| \leqq|| f_{m}\left\|_{0}|| g_{i}-f_{i}\right\|_{0}+\left|\left(f_{m}, f_{j}\right)\right|
$$

it follows from the induction hypothesis and (3.13) that

$$
\left|\left(f_{m}, g_{i}\right)\right| \leqq\left(2 C_{m-1}+1\right) M
$$

for $j<m$ and hence, from (3.14), 


$$
\left\|f_{m}-\tilde{g}_{m}\right\|_{0} \leqq(m-1)^{1 / 2}\left(2 C_{m-1}+1\right) M .
$$

Now, since $g_{m}=\tilde{g}_{m} /\left\|\tilde{g}_{m}\right\|_{0}$,

$$
\begin{aligned}
|| \tilde{g}_{m}-g_{m} \|_{0} & =||\left|\tilde{g}_{m} \|_{0}-1\right| \\
& \leqq||\left|\tilde{g}_{m}\left\|_{0}-|| f_{m}||_{0}|+||| f_{m}\right\|_{0}-1\right| \\
& \leqq|| \tilde{g}_{m}-f_{m}||_{0}+||\left|f_{m} \|_{0}-1\right| \\
& \leqq\left[(m-1)^{1 / 2}\left(2 C_{m-1}+1\right)+1\right] M
\end{aligned}
$$

For $M \leqq 1$ the result follows from (3.15), (3.16) and the triangle inequality. If $M>1$ we have

$$
\left\|f_{m}-g_{m}\right\|_{0} \leqq\left\|f_{m}\right\|_{0}+1 \leqq 2+M \leqq 3 M
$$

and hence the lemma is proved.

We shall also need:

LEMMA 3.5. Let $s \geqq 0$ be given and let $\left\{u_{i}\right\}_{i=1}^{m}$ be an orthonormal set in $\mathfrak{L}_{2}(\Omega)$ such that $u_{j} \in H^{s}(\Omega)$ for each $j$. There exists a constant $C$ such that if $\left\{\tilde{w}_{j}\right\}_{j=1}^{m}$ is any linearly independent set and $\left\{w_{i}\right\}_{i=1}^{m}$ is its Gram-Schmidt orthonormalization then

$$
\max _{1 \leqq k \leqq m}\left\|w_{k}-\tilde{w}_{k}\right\|_{0} \leqq C \max _{1 \leqq i \leqq m}\left(\left\|u_{i}-\tilde{w}_{j}\right\|_{-s}+\left\|u_{i}-\tilde{w}_{i}\right\|_{0}^{2}\right) \text {. }
$$

Proof. We have, from Lemma 3.4, a constant $C$ depending only on $m$ such that

$$
\max _{1 \leqq k \leqq m}|| w_{k}-\tilde{w}_{k} \|_{0} \leqq C \max _{1 \leqq i, j \leqq m}\left|\left(\tilde{w}_{i}, \tilde{w}_{i}\right)-\delta_{i j}\right| .
$$

Now, for fixed $i$ and $j$,

$$
\begin{aligned}
\left|\left(\tilde{w}_{i}, \tilde{w}_{j}\right)-\delta_{i j}\right|= & \left|\left(\tilde{w}_{i}, \tilde{w}_{j}\right)-\left(u_{i}, u_{j}\right)\right| \\
= & \left|\left(\tilde{w}_{i}-u_{i}, \tilde{w}_{i}-u_{i}\right)+\left(u_{i}, \tilde{w}_{j}-u_{i}\right)+\left(\tilde{w}_{i}-u_{i}, u_{i}\right)\right| \\
\leqq & || u_{i}-\tilde{w}_{i}\left\|_{0}\right\| u_{i}-\tilde{w}_{j}\left\|_{0}+\right\| u_{i}\left\|_{s}\right\| u_{i}-\tilde{w}_{i} \|_{-s} \\
& +\left\|u_{i}\right\|_{s}\left\|u_{i}-\tilde{w}_{i}\right\|_{-s} .
\end{aligned}
$$

The result follows easily from (3.17) and (3.18).

We now state and prove the first principal result of this section.

THEOREM 1. Let $\left\{u_{i}\right\}_{i=1}^{m}$ be an orthonormal basis for $R(E(\mu))$ and $s_{1} \geqq 0$. There exist constants $C$ and $h_{2}$ and an orthonormal basis $\left\{w_{i}\right\}_{i=1}^{m}$ for $R\left(E_{h}(\mu)\right)$ such that, for $0 \leqq s \leqq s_{0}$ and $0<h \leqq h_{2}$,

$$
\begin{aligned}
& \max _{1 \leqq k \leqq m}\left\|u_{k}-w_{k}\right\|_{-s} \\
& \quad \leqq C\left\{\left\|T-T_{h}\right\|_{-s_{, s_{1}}}+\left\|T-T_{h}\right\|_{-s, 0}\left\|T-T_{h}\right\|_{0, s_{1}}+\left\|T-T_{h}\right\|_{0, s_{1}}^{2}\right\} .
\end{aligned}
$$

Proof. Let $\left\{\tilde{w}_{j}\right\}_{i=1}^{m}$ be the basis for $R\left(E_{h}(\mu)\right)$ given by Lemma 3.3 and $\left\{w_{i}\right\}_{i=1}^{m}$ its Gram-Schmidt orthonormalization. Then

$$
\left\|u_{k}-w_{k}\right\|_{-s} \leqq\left\|u_{k}-\tilde{w}_{k}\right\|_{-s}+\left\|\tilde{w}_{k}-w_{k}\right\|_{0} .
$$

Applying Lemma 3.5 we obtain, for an appropriate constant $C$,

$$
\left\|w_{k}-\tilde{w}_{k}\right\|_{0} \leqq C \max _{1 \leqq i \leqq m}\left(\left\|u_{j}-\tilde{w}_{j}\right\|_{-s}+\left\|u_{j}-\tilde{w}_{j}\right\|_{0}^{2}\right) .
$$


The theorem now follows by using the estimate (3.11) on the right-hand side of (3.20).

We may also start with an orthonormal basis in $R\left(E_{h}(\mu)\right)$ and obtain a close orthonormal basis for $R(E(\mu))$. This we see in the following:

COROLlARY 1. For each $h$ with $0<h \leqq h_{2}$ let $\left\{w_{j}\right\}_{i=1}^{m}$ be an orthonormal basis for $R\left(E_{h}(\mu)\right)$. Then there is an orthonormal basis $\left\{u_{i}\right\}_{j=1}^{m}$ for $R(E(\mu))$ such that

$$
\begin{aligned}
\max _{1 \leqq i \leqq m}\left\|u_{i}-w_{i}\right\|_{-s} & \\
& \leqq C\left\{\left\|T-T_{h}\right\|_{-s, s_{1}}+\left\|T-T_{h}\right\|_{-s, 0}\left\|T-T_{h}\right\|_{0, s_{1}}+\left\|T-T_{h}\right\|_{0, s_{1}}^{2}\right\}
\end{aligned}
$$

holds for all $0 \leqq s \leqq s_{0}$, where $C$ is a constant independent of $h$.

Proof. Let $\left\{u_{i}^{\prime}\right\}_{j=1}^{m}$ be an orthonormal basis for $R(E(\mu))$ and let $\left\{w_{i}^{\prime}\right\}_{i=1}^{m}$ be the corresponding orthonormal basis in $R\left(E_{h}(\mu)\right)$ given by Theorem 1 . Now $w_{j}=$ $\sum_{k=1}^{m}\left(w_{i}, w_{k}^{\prime}\right) w_{k}^{\prime}$. Set $u_{j}=\sum_{k=1}^{m}\left(w_{i}, w_{k}^{\prime}\right) u_{k}^{\prime}$. The result follows by applying the estimate of Theorem 1 to each term on the right-hand side of the expression $u_{i}-w_{i}=$ $\sum_{k=1}^{m}\left(w_{i}, w_{k}^{\prime}\right)\left(u_{k}^{\prime}-w_{k}^{\prime}\right)$.

Theorem 1 and Corollary 1 show that the "gap" between $R\left(E_{h}(\mu)\right)$ and $R(E(\mu))$ is estimated by the right-hand side of (3.19) (cf. [25]).

We want next to study the eigenvalues of $T_{h}$ which are inside $\Gamma$ and their relationship to $\mu$. As before, let $\mu_{1}(h), \cdots, \mu_{m}(h)$ denote the eigenvalues of $T_{h}$ which lie inside $\Gamma$. In case they are not all distinct, they are counted according to their algebraic multiplicities. Now, it is well known that the individual $\mu_{j}(h)$ 's may be rather poor approximations to $\mu$ because of the nonselfadjointness of $T$ although by Lemma 2.2 they converge to $\mu$ as $h \rightarrow 0$. However, their arithmetic mean is a much better approximation to $\mu$ (cf. [25]). We therefore define

$$
\hat{\mu}(h)=\frac{1}{m} \sum_{i=1}^{m} \mu_{i}(h) .
$$

The next lemma gives representations for $\mu$ and $\hat{\mu}(h)$ in terms of $T, T_{h}$ and elements of the subspaces $R(E(\mu))$ and $R\left(E_{h}(\mu)\right.$ ).

LEMMA 3.6. Let $\left\{u_{i}\right\}_{i=1}^{m}$ be any orthonormal basis for $R(E(\mu))$ and $\left\{w_{i}\right\}_{i=1}^{m}$ be any orthonormal basis for $R\left(E_{h}(\mu)\right)$ with $0<h \leqq h_{0}$. Then

$$
\mu=\frac{1}{m} \sum_{i=1}^{m}\left(T u_{i}, u_{i}\right)
$$

and

$$
\hat{\mu}(h)=\frac{1}{m} \sum_{i=1}^{m}\left(T_{h} w_{i}, w_{j}\right) .
$$

Proof. Denote by $\tilde{T}$ the restriction of $T$ to $R(E(\mu))$ and by $\tilde{T}_{h}$ the restriction of $T_{h}$ to $R\left(E_{h}(\mu)\right)$. We first observe, since $R(E(\mu))$ is an invariant subspace for $T$, that $\sigma(\tilde{T})=\{\mu\}$ and that $\mu$ is an eigenvalue of $\tilde{T}$ with algebraic multiplicity $m$. Similarly, for $0<h \leqq h_{0}, \sigma\left(\tilde{T}_{h}\right)=\left\{\mu_{1}(h), \cdots, \mu_{m}(h)\right\}$. Since on the one hand trace $\tilde{T}=m \mu$ and on the other hand trace $\tilde{T}$ is equal to the sum of the diagonal elements of any one of its matrix representations, (3.21) follows. In the same way we obtain (3.22).

We are now in a position to state and prove the second main result of this section.

THEOREM 2. Let $s_{1}$ be a given nonnegative real number. Then there exist constants $C$ and $h_{2}$ such that, for $0<h \leqq h_{2}$, 


$$
\begin{aligned}
& |\mu-\hat{\mu}(h)| \\
& \quad \leqq C\left\{\left\|T-T_{h}\right\|_{-s_{0}, s_{1}}+\left\|T-T_{h}\right\|_{-s_{0}, 0}\left\|T-T_{h}\right\|_{0, s_{1}}+\left\|T-T_{h}\right\|_{0_{, s_{1}}}^{2}\right\} .
\end{aligned}
$$

Proof. Let $\left\{u_{i}\right\}_{i=1}^{m}$ be any orthonormal basis for $R(E(\mu))$ and let $\left\{w_{i}\right\}_{i=1}^{m}$ be the corresponding orthonormal basis for $R\left(E_{h}(\mu)\right)$ given by Theorem 1, Then, by Lemma 3.6, we have

$$
\mu-\hat{\mu}(h)=\frac{1}{m} \sum_{j=1}^{m}\left[\left(T u_{i}, u_{i}\right)-\left(T_{h} w_{i}, w_{i}\right)\right] .
$$

We will obtain the estimate (3.23) by re-expressing the right-hand side of (3.24): Then

$$
\begin{aligned}
(T u, u)-\left(T_{h} w, w\right)= & (T(u-w), u)-\left(\left(T-T_{h}\right)(u-w), u\right) \\
& +(T u, u-w)-\left(\left(T-T_{h}\right) u, u-w\right) \\
& +\left(\left(T-T_{h}\right) u, u\right)-\left(T_{h}(u-w), u-w\right) .
\end{aligned}
$$

We estimate the terms on the right-hand side of (3.25) as follows:

$$
\begin{aligned}
& |(T(u-w), u)| \leqq\left\|T||_{-s_{0},-s_{0}}\right\| u-w\left\|\left.\right|_{s_{0}}\right\| u \|_{s_{0}}, \\
& \left|\left(\left(T-T_{h}\right)(u-w), u\right)\right| \leqq\left\|T-T_{h}\right\|_{-_{s_{0}, 0}}\|u-w\|_{0}\|u\|_{s_{0}}, \\
& |(T u, u-w)| \leqq\|T\|_{s_{0}, s_{0}}\|u\|_{s_{0}}\|u-w\|_{s_{0}}, \\
& \left|\left(\left(T-T_{h}\right) u, u-w\right)\right| \leqq\left\|T-T_{h}\right\|_{0_{s_{1}}}|| u\left\|_{s_{1}}|| u-w\right\|_{0}, \\
& \left|\left(\left(T-T_{h}\right) u, u\right)\right| \leqq\left\|T-T_{h}\right\|_{-s_{0}, s_{1}}\|u\|_{s_{1}}\|u\|_{s_{0}}, \\
& \left|\left(T_{h}(u-w), u-w\right)\right| \leqq\left\|T_{h}\right\|_{0,0}\|u-w\|_{0}^{2} .
\end{aligned}
$$

Since $\left\|T-T_{h}\right\|_{0,0} \rightarrow 0$ as $h \rightarrow 0,\left\|T_{h}\right\|_{0,0}$ is bounded uniformly in $h$ for $0<h \leqq h_{2}$. Now each expression on the right-hand side of each of the six inequalities is bounded by a constant times the right-hand side of (3.19) with $s=s_{0}$. Hence, using these estimates with (3.25) and (3.24), the proof of Theorem 2 is complete.

We may also estimate $\mu-\mu_{k}(h)$ for each $k$.

THEOREM 3. Let $\alpha$ be the ascent of $\mu-T$. Then there are constants $C$ and $h_{2}$ such that, for $0<h \leqq h_{2}$ and $s_{1} \geqq 0$,

$$
\begin{aligned}
\max _{1 \leqq k \leqq m} \mid \mu & -\left.\mu_{k}(h)\right|^{\alpha} \\
& \leqq C\left\{\left\|T-T_{h}\right\|_{-s_{0}, s_{1}}+\left\|T-T_{h}\right\|_{-s_{0}, 0}\left\|T-T_{h}\right\|_{0, s_{1}}+\left\|T-T_{h}\right\|_{0, s_{1}}^{2}\right\} .
\end{aligned}
$$

Proof. Let $w$ be a unit eigenfunction corresponding to $\mu_{k}(h)$. By Corollary 1 there is a unit vector $u \in R(E(\mu))$ with $\|u-w\|_{-s}$ estimated by the right-hand side of (3.19). Now, noting that $(\mu-T)^{\alpha} u=0$, we have

$$
\begin{aligned}
\left|\mu-\mu_{k}(h)\right|^{\alpha} & =\left|\left(\left[\left(\mu-\mu_{k}(h)\right)^{\alpha}-(\mu-T)^{\alpha}\right] u, u\right)\right| \\
& =\left|\sum_{i=0}^{\alpha-1}\left(\mu-\mu_{k}(h)\right)^{i}\left((\mu-T)^{\alpha-1-j}\left(\mu_{k}(h)-T\right) u, u\right)\right| .
\end{aligned}
$$

Since $R(E(\mu))$ consists of generalized eigenfunctions, $\|u\|_{s}$ will be bounded in $h$ for 
any fixed $s$ even though $u$ may depend on $h$. Using this and the fact that $T$ is bounded in $H^{-s}(\Omega)$ we obtain

$$
\left|\mu-\mu_{k}(h)\right|^{\alpha} \leqq C\left\|\left(\mu_{k}(h)-T\right) u\right\|_{-s_{0}} .
$$

Adding and subtracting $w$ and observing that $\left(\mu_{k}(h)-T_{h}\right) w=0$ gives

$$
\begin{aligned}
& \left|\mu-\mu_{k}(h)\right|^{\alpha} \\
& \quad \leqq C\left\{\left.\left\|\left(\mu_{k}(h)-T\right)(u-w)\right\|\right|_{-s_{0}}+\left\|\left(T-T_{h}\right)(u-w)\right\|_{-s_{0}}+\left\|\left(T-T_{h}\right) u\right\|_{-s_{0}}\right\}
\end{aligned}
$$

from which we obtain

$$
\left|\mu-\mu_{k}(h)\right|^{\alpha} \leqq C\left\{\|u-w\|_{-s_{0}}+\left\|T-T_{h}\right\|_{-s_{0}, 0}\|u-w\|_{0}+\left\|T-T_{h}\right\|_{-s_{0}, s_{1}}\right\} .
$$

The result follows immediately from Corollary 1 .

Finally in this section we obtain quite easily results concerning the proximity of certain elements of $R\left(E_{h}(\mu)\right)$ to certain elements of $R(E(\mu))$. For example, for small $h$ an eigenvector in $R\left(E_{h}(\mu)\right)$ will be close to an eigenvector in $R(E(\mu))$. The full result is given in the following:

THEOREM 4. Let $\mu(h)$ be an eigenvalue of $T_{h}$ such that $\mu(h) \rightarrow \mu$ as $h \rightarrow 0$ and $s_{1} \geqq 0$. Suppose, for each $h, w$ is a unit vector satisfying $\left(\mu(h)-T_{h}\right)^{k} w=0$ for some positive integer $k \leqq \alpha$. Then, for any integer $l$ with $k \leqq l \leqq \alpha$, there is a vector $u \in$ $R(E(\mu))$ such that $(\mu-T)^{l} u=0$ and

$$
\|u-w\|_{0} \leqq C\left\|T-T_{h}\right\|_{0, s_{1}}^{(l-k+1) / \alpha} .
$$

Proof. From the Riesz-Schauder theory and the closed graph theorem it follows that $\left(\mu-\left.T\right|_{\mathscr{L}_{2}(\Omega)}\right)^{l}$ has a bounded right inverse which maps $R\left(\left(\mu-\left.T\right|_{\mathcal{L}_{2}(\Omega)}\right)^{l}\right)$ to the orthogonal complement of $\mathfrak{N}(\mu-T)^{l}$ in $\mathfrak{L}_{2}(\Omega)$. Thus, setting $u=P w$ where $P$ is the orthogonal projection of $\mathscr{L}_{2}(\Omega)$ onto $\Re\left((\mu-T)^{l}\right)$, there is a constant $C$ independent of $w$ such that

$$
\|w-u\|_{0} \leqq C\left\|(\mu-T)^{l}(w-u)\right\|_{0} .
$$

By Corollary 1 there is a unit vector $\tilde{u}$ in $R(E(\mu))$ such that

$$
\|w-\tilde{u}\|_{0} \leqq C\left\|T-T_{h}\right\|_{0, s_{1}} .
$$

Hence

$$
\begin{aligned}
\|\left[(\mu-T)^{l}\right. & \left.-\left(\mu-T_{h}\right)^{l}\right] w \|_{0} \\
& =\left\|\sum_{j=0}^{l-1}\left(\mu-T_{h}\right)^{i}\left(T_{h}-T\right)(\mu-T)^{l-i-1}[(w-\tilde{u})+\tilde{u}]\right\|_{0} \\
& \leqq C\left(\|w-\tilde{u}\|_{0}+\left\|T_{h}-T\right\|_{0, s_{1}}\right) \leqq C\left\|T_{h}-T\right\|_{0, s_{1}} .
\end{aligned}
$$

Since $k \leqq l$,

$$
\begin{aligned}
\left\|\left(\mu-T_{h}\right)^{l} w\right\|_{0} & =\left\|\sum_{i=0}^{l}\left(\begin{array}{l}
l \\
j
\end{array}\right)(\mu-\mu(h))^{i}\left(\mu(h)-T_{h}\right)^{l-i} w\right\|_{0} \\
& =\left\|\sum_{i=l-k+1}^{l}\left(\begin{array}{l}
l \\
j
\end{array}\right)(\mu-\mu(h))^{i}\left(\mu(h)-T_{h}\right)^{l-i} w\right\|_{0} \\
& \leqq C|\mu-\mu(h)|^{l-k+1} .
\end{aligned}
$$


Using (3.26), (3.27), and (3.28), we have

$$
\begin{aligned}
\|w-u\|_{0} & \leqq C\left\|\left[(\mu-T)^{l}-\left(\mu-T_{h}\right)^{l}\right] w+\left(\mu-T_{h}\right)^{l} w\right\|_{0} \\
& \leqq C\left\{|\mu-\mu(h)|^{l-k+1}+\left\|T_{h}-T\right\|_{0, s_{1}}\right\} .
\end{aligned}
$$

The result now follows from Theorem 3 .

4. Second-Order Boundary Value Problems. Let $L$ be a second-order operator given by

$$
L \varphi=-\sum_{i, j=1}^{x} \frac{\partial}{\partial x_{i}}\left(a_{i j} \frac{\partial \varphi}{\partial x_{j}}\right)+\sum_{i=1}^{x} b_{i} \frac{\partial \varphi}{\partial x_{i}}+c \varphi
$$

where $a_{i j}, b_{i}$ and $c$ are in $C^{\infty}(\bar{\Omega})$ and $a_{i j}=a_{j i}$. We will assume that $L$ is uniformly strongly elliptic; i.e., there is a positive constant $a_{0}$ such that

$$
\operatorname{Re} \sum_{i, j=1}^{N} a_{i j}(x) \xi_{i} \xi_{j} \geqq a_{0} \sum_{i=1}^{x} \xi_{i}^{2}
$$

for all real $\xi_{1}, \cdots, \xi_{N}$ and all $x \in \Omega$. The sesquilinear form on $H^{1}(\Omega) \times H^{1}(\Omega)$ associated with $L$ is given by

$$
B(\varphi, \psi)=\sum_{i, j=1}^{N} \int_{\Omega} a_{i j} \frac{\partial \varphi}{\partial x_{i}} \frac{\partial \bar{\psi}}{\partial x_{j}} d x+\sum_{i=1}^{N} \int_{\Omega} b_{i} \frac{\partial \varphi}{\partial x_{i}} \bar{\psi} d x+\int_{\Omega} c \varphi \bar{\psi} d x
$$

Let $b=\max _{1 \leqq i \leqq N ; x \in \Omega}\left|b_{i}(x)\right|$.

We shall assume without loss that $\operatorname{Re} c \geqq a_{0} / 2+b^{2} / 2 a_{0}$ since adding a constant to $c$ only shifts the eigenvalues. Under this assumption $B$ is coercive on $H^{1}(\Omega)$; i.e.,

$$
\operatorname{Re} B(\varphi, \varphi) \geqq \frac{1}{2} a_{0}\|\varphi\|_{1}^{2}
$$

for all $\varphi \in H^{1}(\Omega)$. Clearly, because of the boundedness of the coefficients, we also have that $B$ is continuous on $H^{1}(\Omega) \times H^{1}(\Omega)$; i.e., for a suitable constant $a_{1}$,

$$
|B(\varphi, \psi)| \leqq a_{1}\|\varphi\|_{1}\|\psi\|_{1}
$$

for all $\varphi, \psi \in H^{1}(\Omega)$.

Let $H_{0}^{1}(\Omega)$ be the closure in $H^{1}(\Omega)$ of the infinitely differentiable functions with compact support in $\Omega, C_{0}^{\infty}(\Omega)$, and $V$ any closed subspace of $H^{1}(\Omega)$ with $H_{0}^{1}(\Omega) \subset$ $V \subset H^{1}(\Omega)$. A class of boundary value problems associated with $B$ may be formulated as follows: Given $f \in \mathfrak{L}_{2}(\Omega)$ find $u \in V$ such that for all $\varphi \in V$,

$$
B(u, \varphi)=(f, \varphi) .
$$

With our assumption on $B$ this problem always has a unique solution $u$ (cf. [26]) and we denote the linear operator which takes $f$ to $u$ by $T$. In the case $V=H_{0}^{1}(\Omega)$ this is just the weak formulation of the Dirichlet problem and for $V=H^{1}(\Omega), T$ is the solution operator for the Neumann problem. We suppose now that $V$ is either $H_{0}^{1}(\Omega)$ or $H^{1}(\Omega)$.

If, in addition, we have $f \in H^{s}(\Omega)$ for $s$ real, the following estimate is valid:

$$
\|T f\|_{s+2} \leqq C_{s}\|f\|_{s}
$$

where $C_{s}$ is an appropriate constant (cf. [35], [36]). The estimate (4.4) allows us to extend the operator $T$ from $\mathfrak{L}_{2}(\Omega)$ to $H^{s}(\Omega)$ for any $s<0$. Now let $s_{0} \geqq 0$ be fixed 
and consider the operator $T$ as being defined on $H^{-s}{ }^{\circ}(\Omega)$. Then (4.4) shows that

$$
T: H^{s}(\Omega) \rightarrow H^{s+2}(\Omega)
$$

as a bounded operator for $-s_{0} \leqq s$. Hence $T$ as defined here satisfies the conditions set forth in Section 3.

An eigenvalue corresponding to the boundary value problem is a complex number $\lambda$ such that

$$
B(u, \varphi)=\lambda(u, \varphi)
$$

for some nonzero $u \in V$ and all $\varphi \in V$. An eigenvalue of $T$ is a complex number $\mu$ such that $T u=\mu u$ for some nonzero $u \in H^{-s o}(\Omega)$. As was pointed out above, the spectrum of $T$ consists of nonzero eigenvalues and zero. Clearly, for any eigenvalue $\mu$ of $T$ we have that $\lambda=1 / \mu$ is an eigenvalue satisfying (4.5). Similarly, for $\lambda$ an eigenvalue of (4.5), $\mu=1 / \lambda$ is an eigenvalue of $T$. It is also well known, because of the smoothness assumptions on the coefficients, that $L u=\lambda u$ in $\Omega$ and, depending on the choice of $V, u$ will satisfy certain boundary contitions. For $V=H_{0}^{1}(\Omega)$ we have

$$
u=0 \text { on } \partial \Omega
$$

and for $V=H^{1}(\Omega)$,

$$
\partial u / \partial \nu=0 \text { on } \partial \Omega
$$

where $\partial / \partial \nu=\sum_{i, j=1}^{v} a_{i} n_{j} \partial / \partial x_{i}$ and $n_{j}$ is the $j$ th component of the outward unit normal on $\partial \Omega$.

Finally, we shall need in our applications some information about the $\mathscr{L}_{2}(\Omega)$ adjoint $T^{*}$ of $\left.T\right|_{\mathcal{L}_{2}(\Omega)}$. This operator is defined by

$$
(T f, \psi)=\left(f, T^{*} \psi\right)
$$

for all $f, \psi \in \mathscr{L}_{2}(\Omega)$ and satisfies $T^{*} \psi \in V$ and $B\left(\varphi, T^{*} \psi\right)=(\varphi, \psi)$ for all $\varphi \in V$.

In addition we have as for $T$, the regularity estimate for $s \geqq 0$ and $\psi \in H^{s}(\Omega)$,

$$
\left\|T^{*} \psi\right\|_{s+2} \leqq C_{s}^{*}\|\psi\|_{s}
$$

with $C_{s}^{*}$ an appropriate constant. We shall not need (4.6) with $s<0$ even though it is valid.

5. Finite-Dimensional Subspaces of $H^{k}(\Omega)$. Let $\left\{S_{h}\right\}_{0<h \leqq 1}$ be a one-parameter family of finite-dimensional vector spaces. For given integers $k$ and $r$ with $0 \leqq k \leqq r$ we shall say that $\left\{S_{h}\right\}_{0<h \leqq 1}$ is of class $S_{k, r}$ if $S_{h} \subset H^{k}(\Omega)$ for each $h$ and if there is a constant $C$ independent of $h$ such that for any $v \in H^{t}(\Omega)$ with $k \leqq t \leqq r$,

$$
\inf _{\chi \in S_{h}} \sum_{j=0}^{k} h^{j}\|v-\chi\|_{i} \leqq C h^{t}\|v\|_{t}
$$

Analogously, we define the class $\stackrel{\circ}{S}_{k, r}$ to consist of those families $\left\{S_{h}\right\}_{0<h \leqq 1}$ such that for each $h, S_{h} \subset H^{k}(\Omega)$, and (5.1) is required to hold only for $v \in H^{t}(\Omega) \cap H_{0}^{1}(\Omega)$.

In the definition of $S_{k, r}$, condition $(5.1)$ can be replaced by the condition that the same inequality be required to hold for $t=r$ only. This was proved in [12]. 
We note that there are many examples of families of class $\S_{k, r}$. In particular the restriction to $\Omega$ of many different families of piecewise polynomial functions are of class $S_{k, r}$ for certain values of $k$ and $r$ (cf. [17], [23], [24], [39], [54], [55]). Each of the usual sets of trial functions used in conjunction with the finite element method (cf. [53]) is of class $S_{k, r}$ for some $k$ and $r$. The simplest example of these for $N=2$ is the family of restrictions to $\Omega$ of piecewise linear functions on a family of uniform triangulations of the plane with $h$ the diameter of each triangle. Such a family is of class $\delta_{1,2}$ and hence satisfies (5.1) with $k=1$ and $r=2$.

Finally, by using tensor products of $B$-splines [37] it is easy to see that for any number of dimensions and any given values of $k$ and $r$, families of class $S_{k, r}$ may be constructed. For such considerations, cf. [4], [5], [13], [14], [23].

6. Standard Galerkin Methods for the Dirichlet and Neumann Problems. For $V=H^{1}(\Omega)$ let $\left\{S_{h}\right\}_{0<h \leqq 1}$ be a family of spaces of class $\delta_{1, r}$. For $V=H_{0}^{1}(\Omega)$, let $\left\{S_{h}\right\}_{0<h \leqq 1}$ belong to $S_{1, r}$ and, in addition, assume that for each $h, S_{h} \subset H_{0}^{1}(\Omega)$. We note that such families may be difficult to construct in practice for domains of general shape. Hence we will treat Dirichlet's problem again in the later sections by alternative techniques which do not require that $S_{h} \subset H_{0}^{1}(\Omega)$.

We want to consider the eigenvalues of the Galerkin problem; i.e., the complex numbers $\lambda(h)$ satisfying $B(w, \varphi)=\lambda(h)(w, \varphi)$ for some nonzero $w \in S_{h}$ and all $\varphi \in S_{h}$. These we want to consider as approximations to the eigenvalues of (4.5).

To this end we define now a family of operators $\left\{T_{h}\right\}_{0<h \leqq 1}$ on $\mathscr{L}_{2}(\Omega)$ as follows: For each $h$ set $T_{h} f=u_{h}$, where $u_{h}$ is the unique solution in $S_{h}$ of the equation

$$
B\left(u_{h}, \varphi\right)=(f, \varphi)
$$

for all $\varphi \in S_{h}$. The inequality (4.1) guarantees the existence and uniqueness of $u_{h}$. Hence, for each $h$,

$$
T_{h}: \mathscr{L}_{2}(\Omega) \rightarrow S_{h} \subset \mathscr{L}_{2}(\Omega)
$$

and, since $S_{h}$ is finite-dimensional, $T_{h}$ is compact.

Let $\mu(h)$ be a nonzero eigenvalue of $T_{h}$. Then there is a nonzero element $w$ of $S_{h}$ such that $T_{h} w=\mu(h) w$. By the definition of $T_{h}$,

$$
B(w, \varphi)=\frac{1}{\mu(h)} B\left(T_{h} w, \varphi\right)=\frac{1}{\mu(h)}(w, \varphi)
$$

so that $\lambda(h)=1 / \mu(h)$ is an eigenvalue of the Galerkin problem. Conversely, since none of the eigenvalues $\lambda(h)$ can be zero, each $\mu(h)=1 / \lambda(h)$ is an eigenvalue of $T_{h}$. Hence we may compare the eigenvalues of (4.5) with the Galerkin approximations by comparing the eigenvalues of $T$ with those of $T_{h}$.

In order to apply our theorems we shall obtain estimates for $T-T_{h}$. Let $f \in$ $\mathfrak{L}_{2}(\Omega)$ be arbitrary. Combining (4.3) and (6.1) we have that

$$
B\left(\left(T-T_{h}\right) f, \varphi\right)=0
$$

for all $\varphi \in S_{h}$. Hence, using (4.1) and (6.2), it follows that

$$
\begin{aligned}
\left\|\left(T-T_{h}\right) f\right\|_{1}^{2} & \leqq 2 / a_{0}\left|B\left(\left(T-T_{h}\right) f,\left(T-T_{h}\right) f\right)\right| \\
& =2 / a_{0}\left|B\left(\left(T-T_{h}\right) f, T f-\chi\right)\right|
\end{aligned}
$$


for any $\chi \in S_{h}$. Using the continuity of $B$, (4.2), it follows that for a suitable constant $C$,

$$
\left\|\left(T-T_{h}\right) f\right\|_{1} \leqq C \inf _{\chi \in S_{h}}\|T f-\chi\|_{1} .
$$

Now suppose that $f \in H^{t-2}(\Omega)$ for some $t$ with $2 \leqq t \leqq r$. Then by (5.1) we have that

$$
\left\|\left(T-T_{h}\right) f\right\|_{1} \leqq C h^{t-1}\|T f\|_{t} .
$$

But for $f \in H^{t-2}(\Omega)$,

$$
\|T f\|_{t} \leqq C\|f\|_{t-2}
$$

where we have used the estimate (4.4) with $s=t-2$. Combining (6.3) and (6.4), it follows that

$$
\left\|\left(T-T_{h}\right) f\right\|_{1} \leqq C h^{t-1}\|f\|_{t-2}
$$

for $2 \leqq t \leqq r$.

Now we want to estimate norms of $\left(T-T_{h}\right) f$ with index lower than 1 . To this end let $\psi \in C^{\infty}(\bar{\Omega})$ be arbitrary. We have that

$$
\left(\left(T-T_{h}\right) f, \psi\right)=B\left(\left(T-T_{h}\right) f, T^{*} \psi\right)=B\left(\left(T-T_{h}\right) f, T^{*} \psi-\chi\right)
$$

for any $\chi \in S_{h}$. Using (4.2) and (6.5) we obtain from (6.6),

$$
\left|\left(\left(T-T_{h}\right) f, \psi\right)\right| \leqq C h^{t-1}\|f\|_{t-2}\left\|T^{*} \psi-\chi\right\|_{1} .
$$

Now since $\chi$ is arbitrary we may take the infimum on the right-hand side of (6.7) and use (5.1) with $t=s+2,0 \leqq s \leqq r-2$ to obtain

$$
\left|\left(\left(T-T_{h}\right) f, \psi\right)\right| \leqq C h^{s+t}\|f\|_{t-2} \| T^{*} \psi||_{s+2} .
$$

Using the estimate (4.6) we have that

$$
\left|\left(\left(T-T_{h}\right) f, \psi\right)\right| \leqq C h^{s+t}\|f\|_{t-2}\|\psi\|_{s}
$$

for $0 \leqq s \leqq r-2$ and $2 \leqq t \leqq r$. Since $\psi$ is arbitrary in $C^{\infty}(\bar{\Omega})$ it follows that

$$
\left\|\left(T-T_{h}\right) f\right\|_{-s}=\sup _{\psi \in C^{\infty}(\bar{\Omega})}\left|\left(\left(T-T_{h}\right) f, \psi\right)\right| /\|\psi\|_{s} \leqq C h^{s+t}\|f\|_{t-2} .
$$

Now dividing by $\|f\|_{t-2}$ and taking the supremum over functions $f \in H^{t-2}(\Omega)$ we obtain

$$
\left\|\left(T-T_{h}\right)\right\|_{-s, t-2} \leqq C h^{s+t}
$$

for $0 \leqq s \leqq r-2$ and $2 \leqq t \leqq r$.

If we take $s=0$ and $t=2$ in (6.8) we obtain easily that (3.2) is satisfied. Hence Theorems 1-4 apply with $s_{0}=s_{1}=r-2$. Thus let $\lambda$ be any eigenvalue with algebraic multiplicity $m$ satisfying (4.5) and $\mu=1 / \lambda$. Then the eigenvalues $\mu_{1}(h), \cdots, \mu_{m}(h)$ which converge to $\mu$ as $h \rightarrow 0$ are computed as the reciprocals of certain eigenvalues $\lambda_{1}(h), \cdots, \lambda_{m}(h)$ of the finite-dimensional eigenvalue problem

$$
B(w, \varphi)=\lambda_{i}(h)(w, \varphi)
$$

for nonzero $w \in S_{h}$ and all $\varphi \in S_{h}$. Theorem 2 thus yields the estimate 


$$
\left|\lambda-\left(\frac{1}{m} \sum_{i=1}^{m} 1 / \lambda_{j}(h)\right)^{-1}\right| \leqq C h^{2 r-2} .
$$

We point out that in the case of a simple eigenvalue $(m=1)$ we have that $|\lambda-\lambda(h)| \leqq C h^{2 r-2}$, and that this estimate yields the same rate of convergence obtainable in the case that the problem is selfadjoint using the methods of [11].

Using Theorem 1 with $s=0$ we obtain for the invariant subspaces

$$
\max _{1 \leqq i \leqq m}\left\|u_{j}-w_{j}\right\|_{0} \leqq C h^{r} .
$$

We note that in case the boundary or the coefficients do not possess the required smoothness properties we would obtain lower rates of convergence. In such a case this would indeed be expected.

7. Least Squares Method. One method of approximating the solution of the Dirichlet problem using subspaces $S_{h}$ which are not required to satisfy any boundary conditions is the least squares method of Bramble and Schatz [15], [16]. Although the case studied in [15] was that of real coefficients all relevant theorems from [15] are valid in the case of strongly elliptic operators with complex coefficients with the spaces taken to be complex.

Now let $\left\{S_{h}\right\}_{0<h \leqq 1}$ be of class $S_{2, r}$ with $r \geqq 4$. Then the least squares approximation to the solution of Dirichlet's problem is given as follows: For $f \in \mathfrak{L}_{2}(\Omega)$ define $u_{h}$ to be the unique element of $S_{h}$ such that

$$
\left\|L_{h}-f\right\|_{0}^{2}+h^{-3}\left|u_{h}\right|^{2}=\inf _{\chi \in S_{h}}\left\{\|L \chi-f\|_{0}^{2}+h^{-3}|\chi|^{2}\right\} .
$$

Here we have used the notation

$$
|\varphi|^{2}=\int_{\partial \Omega}|\varphi|^{2} d \sigma
$$

with $\sigma$ the measure on $\partial \Omega$ induced by the Lebesgue measure on $\mathrm{R}^{N-1}$. The corresponding inner product will henceforth be denoted by

$$
\langle\varphi, \psi\rangle=\int_{\partial \Omega} \varphi \bar{\psi} d \sigma
$$

Because of uniqueness in the Dirichlet problem, (4.3) with $V=H_{0}^{1}(\Omega), u_{h} \in S_{h}$ exists and is unique and we denote the corresponding linear solution operator by $T_{h}$. Hence we have that $T_{h} f=u_{h}$ and $T_{h}: \mathscr{L}_{2}(\Omega) \rightarrow S_{h} \subset \mathscr{L}_{2}(\Omega)$ and is compact.

Clearly, an equivalent characterization of $u_{h}$ is given by

$$
\left(L u_{h}, L \varphi\right)+h^{-3}\left\langle u_{h}, \varphi\right\rangle=(f, L \varphi)
$$

for all $\varphi \in S_{h}$. Now it is easy to see that $\mu(h)$ is a nonzero eigenvalue of $T_{h}$ and $w \in S_{h}$ is a corresponding eigenfunction if and only if

$$
(L w, L \varphi)+h^{-3}\langle w, \varphi\rangle=(w, L \varphi) / \mu(h)
$$

for all $\varphi \in S_{h}$. Hence, again, we may compare the eigenvalues $\lambda(h)=1 / \mu(h)$ of the system (7.1) with those of (4.5) by comparing the eigenvalues of $T$ with those of $T_{h}$.

Note that even in the case that $L$ is selfadjoint, (7.1) gives rise to a nonsymmetric 
matrix eigenvalue problem so that the convergence estimates for the eigenvalues do not follow from the standard techniques using the Rayleigh quotient. In particular, the technique of [11] does not apply.

In order to see that Theorems 1-4 apply, we need only give estimates for $T-T_{h}$ in various norms. But these follow directly from Corollary 4.1 of [15]. In Corollary 4.1, take $\gamma=\frac{3}{2}, \lambda=t-2, g \equiv 0$ and $l=-s$. We have $e=\left(T-T_{h}\right) f$ and $\left\|\left(T-T_{h}\right) f\right\|_{-s} \leqq C h^{s+t}\|f\|_{t-2}$ for $0 \leqq s \leqq r-4$ and $2 \leqq t \leqq r$. This means that $\left\|\left(T-T_{h}\right)\right\|_{-s, t-2} \leqq C h^{s+t}$ for $0 \leqq s \leqq r-4$ and $2 \leqq t \leqq r$. Taking $s=0$ and $t=2$ we see immediately that (3.2) is satisfied. Now let $\lambda$ be an eigenvalue of (4.5) with algebraic multiplicity $m$. Then the $m$ eigenvalues $\lambda_{1}(h), \cdots, \lambda_{m}(h)$ which converge to $\lambda$ satisfy

$$
\left|\lambda-\left(\frac{1}{m} \sum_{j=1}^{m} 1 / \lambda_{j}(h)\right)^{-1}\right| \leqq C h^{2 r-4} .
$$

This is just the result of Theorem 2 with $s_{0}=r-4$ and $s_{1}=r-2$.

The estimate (6.10) given by Theorem 1 is the same in this case.

8. Nitsche's Method for the Dirichlet Problem. Although the least squares method requires nothing special about the elements of $S_{h}$ at the boundary of $\Omega$, it does involve second derivatives in the quadratic form and hence might lead to problems of conditioning of the resulting linear system.

Nitsche has proposed a method in [28] for approximating the solution of the Dirichlet problem for Poisson's equation. This method involves a quadratic form which is not bounded below on the whole space but which is positive definite on subspaces $S_{h}$ which satisfy certain additional conditions. Because of this indefiniteness property the standard techniques for dealing with selfadjoint problems do not lead to a convergence proof or estimates for the rate of convergence of the corresponding eigenvalues.

Here we shall formulate Nitsche's method for the general nonselfadjoint equation with complex coefficients and prove the estimates necessary for the application of our theorems to the study of eigenvalues.

Let $\left\{S_{h}\right\}_{0<h \leqq 1}$ be of class $\stackrel{\circ}{S}_{2, r}$ and, in addition, we assume that there is a constant $C_{1}$ such that

$$
\sum_{j=1}^{N} \int_{\partial \Omega}\left|\frac{\partial \varphi}{\partial x_{j}}\right|^{2} d \sigma \leqq C_{1} h^{-1}\|\varphi\|_{1}^{2}
$$

for all $\varphi \in S_{h}$ and $0<h \leqq 1$.

Define now the quadratic form

$$
N_{\gamma}(\varphi, \psi)=B(\varphi, \psi)-\left\langle\varphi, \frac{\partial \psi}{\partial \bar{\nu}}-\sum_{j=1}^{N} b_{i} n_{j} \psi\right\rangle-\left\langle\frac{\partial \varphi}{\partial \nu}, \psi\right\rangle+\gamma h^{-1}\langle\varphi, \psi\rangle,
$$

where $\gamma$ is a positive constant, $\partial / \partial \nu=\sum_{i, j=1}^{v} a_{i j} n_{i} \partial / \partial x_{i}$ (as in Section 4) and $\partial / \partial \bar{\nu}=\sum_{i, j=1}^{v} \bar{a}_{i j} n_{i} \partial / \partial x_{j}$.

Let us define, for each $h$, the norm

$$
\|\varphi\| \|=\left(\sum_{j=1}^{N}\left[\left\|\frac{\partial \varphi}{\partial x_{j}}\right\|_{0}^{2}+h\left|\frac{\partial \varphi}{\partial x_{j}}\right|^{2}\right]+h^{-1}|\varphi|^{2}\right)^{1 / 2}
$$


for $\varphi \in H^{2}(\Omega)$ with $\partial \varphi / \partial x_{i}$ on $\partial \Omega$ its trace. It is a simple consequence of (4.1) and Schwarz's inequality that there are constants $C$ and $\gamma_{0}$ such that for $\gamma \geqq \gamma_{0}$,

$$
\||\varphi|\|^{2} \leqq C\left|N_{\gamma}(\varphi, \varphi)\right|
$$

for all $\varphi \in S_{h}$. In this estimate explicit use is made of (8.1).

It is equally clear that $N_{\gamma}$ is a sesquilinear form which is continuous with respect to ||$|\cdot|||$; i.e., there is a constant $C$ such that

$$
\left|N_{\gamma}(\varphi, \psi)\right| \leqq C\|\varphi\|\|\|\|\psi\| \|
$$

for all $\varphi$ and $\psi$ in $H^{2}(\Omega)$. Now for a given $f \in \mathscr{L}_{2}(\Omega)$ let $u_{h}=T_{h} f$ be the unique solution of the equations

$$
N_{\gamma}\left(u_{h}, \varphi\right)=(f, \varphi)
$$

for all $\varphi \in S_{h}$. The existence and uniqueness is assured by (8.2). Thus we have defined a family $\left\{T_{h}\right\}_{0<h \leqq 1}$ of compact operators on $\mathfrak{L}_{2}(\Omega)$. As in the previous sections the corresponding eigenvalues $\lambda(h)$ satisfy

$$
N_{\gamma}(w, \varphi)=\lambda(h)(w, \varphi)
$$

for some nonzero $w$ in $S_{h}$ and all $\varphi \in S_{h}$. Clearly $\mu(h)$ is a nonzero eigenvalue of $T_{h}$ if and only if $\lambda(h)=1 / \mu(h)$ is an eigenvalue of (8.5).

To apply our theorems to the comparison of the eigenvalues of (4.5) with those of (8.5) it again suffices to estimate certain norms of $T-T_{h}$ with $T$ defined through (4.3) with $V=H_{0}^{1}(\Omega)$.

To this end we note first that

$$
N_{\gamma}(T f, \varphi)=(f, \varphi)
$$

for all $\varphi \in H^{2}(\Omega)$. Hence, combining (8.4) and (8.6), we obtain

$$
N_{\gamma}\left(\left(T-T_{h}\right) f, \varphi\right)=0
$$

for all $\varphi \in S_{h}$. We first obtain an estimate for $\left\|\mid\left(T-T_{h}\right) f\right\|$. By the triangle inequality we have that, for any $\chi \in S_{h}$,

$$
\|\|\left(T-T_{h}\right) f\|\| \leqq\|T f-\chi\|\|+\|\left\|\chi-T_{h} f\right\| \| .
$$

Since $\chi-T_{h} f \in S_{h}$, we may use (8.2) to obtain

$$
\begin{aligned}
||\left|\chi-T_{h} f\right|||^{2} & \leqq C\left|N_{\gamma}\left(\chi-T_{h} f, \chi-T_{h} f\right)\right| \\
& =C\left|N_{\gamma}\left(\chi-T f, \chi-T_{h} f\right)\right|,
\end{aligned}
$$

the last equation coming from (8.7). Using (8.9) and (8.3) it follows that

$$
\|\| \chi-T_{h} f\|\mid \leqq C\| \chi \chi-T f\|\| .
$$

The last inequality, $(8.10)$, together with (8.8) yields

$$
\left\|\left(T-T_{h}\right) f\right\|\left\|\leqq C \inf _{\chi \in S_{h}}\right\| \chi-T f\|\| .
$$

Now by standard trace inequalities it follows that for any $v \in H^{2}(\Omega)$,

$$
\left\|\left|v\left\|\mid \leqq C \sum_{j=0}^{2} h^{i-1}\right\| v \|_{i}\right.\right.
$$


for $0<h \leqq 1$. Combining (8.11) and (8.12) and using the property (5.1) of $S_{h}$ we obtain

$$
\|\|\left(T-T_{h}\right) f\|\| \leqq C h^{t-1}\|T f\|_{t}
$$

for $2 \leqq t \leqq r$. It follows then from (4.4) that, if $f \in H^{t-2}(\Omega)$ then

$$
\|\|\left(T-T_{h}\right) f\|\| \leqq C h^{t-1}\|f\|_{\ell-2} .
$$

To estimate the norms $\left\|T-T_{h}\right\|_{-s, t-2}$ for $0 \leqq s \leqq r-2$ and $2 \leqq t \leqq r$ we proceed as in Section 6 . Let $\psi \in C^{\infty}(\bar{\Omega})$ be arbitrary and note that $N_{\gamma}\left(\varphi, T^{*} \psi\right)=$ $(\varphi, \psi)$ for all $\varphi \in H^{2}(\Omega)$. In particular,

$$
\begin{aligned}
\left(\left(T-T_{h}\right) f, \psi\right) & =N_{\gamma}\left(\left(T-T_{h}\right) f, T^{*} \psi\right) \\
& =N_{\gamma}\left(\left(T-T_{h}\right) f, T^{*} \psi-\chi\right)
\end{aligned}
$$

for all $\chi \in S_{h}$. From (8.3) it follows that

$$
\left|\left(\left(T-T_{h}\right) f, \psi\right)\right| \leqq C\left\|\left|\left(T-T_{h}\right) f\right|\right\|\left|\left\|T^{*} \psi-\chi\right\|\right| .
$$

Using (8.13), (8.12) and the approximability assumptions (5.1) it follows that

$$
\left|\left(\left(T-T_{h}\right) f, \psi\right)\right| \leqq C h^{s+t}|| f\left\|_{\ell-2}\right\| T^{*} \psi \|_{s+2} .
$$

The estimate (4.7) yields

$$
\left|\left(\left(T-T_{h}\right) f, \psi\right)\right| \leqq C h^{s+t}\|f\|_{t-2}\|\psi\|_{s},
$$

for $0 \leqq s \leqq r-2$ and $2 \leqq t \leqq r$. This inequality implies immediately that

$$
\left\|T-T_{h}\right\|_{-s, t-2} \leqq C h^{s+t}
$$

for $0 \leqq s \leqq r-2$ and $2 \leqq t \leqq r$. In particular, with $s=0, t=2$, we have

$$
\lim _{h \rightarrow 0}\left\|T-T_{h}\right\|_{0,0}=0
$$

so that Theorems 1-4 apply. The results (6.9) and (6.10) are valid now in this case.

9. Nitsche's Method with Nearly Zero Boundary Conditions. If the subspaces $S_{h}$ satisfy, in addition to condition (8.1), a certain condition of smallness on the boundary then Nitsche [29] has observed that the form $N_{0}$ also leads to good approximation in the Dirichlet problem.

More precisely, suppose that there is a constant $C_{2}$ such that for all $\varphi \in S_{h}$,

$$
|\varphi|^{2} \leqq C_{2} h\|\varphi\|_{1}^{2} .
$$

Suppose further that $C_{2} \leqq\left(a_{0} / 8\right)\left(8 N \tilde{a}^{2} C_{1} / a_{0}+\tilde{b}\right)^{-1}$ where $C_{1}$ is the constant in (8.1) and $\tilde{a}$ and $\tilde{b}$ are constants such that

$$
\max _{1 \leqq i \leqq I ; x \in \partial \Omega}\left|\sum_{i=1}^{N} a_{i j} n_{j}\right| \leqq \tilde{a} \text { and } \max _{x \in \partial \Omega}\left|\sum_{i=1}^{N} b_{i} n_{i}\right| \leqq \tilde{b} .
$$

This condition just says that (9.1) must hold with $C_{2}$ a sufficiently small constant. In fact, in many examples where a condition such as (9.1) is satisfied, it is also satisfied with $C_{2}=C_{2}(h)$ and $C_{2}(h)=o(1)$ as $h \rightarrow 0$. In this connection see [29], [18], [55]. 
Now with this condition satisfied (8.2) is valid with $\gamma=0$ and all subsequent considerations of Section 8 are valid in this case. Hence Theorems 1-4 again apply and the estimates (6.9) and (6.10) are valid in this case.

10. Lagrange Multiplier Method of Babuška. Recently Babuška [6] has introduced a method for constructing an approximate solution of the Dirichlet problem in a subspace $S_{h}$ which belongs to a family of class $S_{1, r}$. He introduces analogous classes of spaces of approximating functions on $\partial \Omega$ and simultaneously obtains an approximation of $\partial u / \partial \nu$, the conormal derivative of the solution on $\partial \Omega$. As in the other examples this method gives rise to a family of operators which fits nicely into our theory for the approximation of eigenvalues. Hence, as we shall see, estimates similar to (6.9) and (6.10) are valid.

In order to describe precisely the method, we need some additional notation. For $s$ any real number let $H^{s}(\partial \Omega)$ denote the Sobolev space of (complex valued) functions on $\partial \Omega$ (cf. [26]) and denote by $|\cdot|_{\text {s }}$ the corresponding norm.

In analogy with Section 5 , let $\left\{S_{h}^{\prime}\right\}_{0<h \leqq 1}$ be a one-parameter family of vector spaces. For given real numbers $k^{\prime}$ and $r^{\prime}$ with $\frac{1}{2} \leqq k^{\prime} \leqq r^{\prime}$ we shall say that $\left\{S_{h}^{\prime}\right\}_{0<h \leqq 1}$ is of class $S_{k^{\prime}, r^{\prime}}^{\prime}$ if $S_{h}^{\prime} \subset H^{k^{\prime}}(\partial \Omega)$ for each $h$ and if there is a constant $C$ independent of $h$ such that for any $v \in H^{s}(\partial \Omega)$ and $-\frac{1}{2} \leqq l \leqq k^{\prime} \leqq s \leqq r^{\prime}$,

$$
\inf _{\chi \in S_{h^{\prime}}}|v-\chi|_{\iota} \leqq C h^{s-l}|v|_{s}
$$

and

$$
|\varphi|_{1 / 2} \leqq C h^{-1}|\varphi|_{-1 / 2}
$$

holds for all $\varphi \in S_{h}^{\prime}$.

An example of a situation in which such a family may be easily constructed is as follows: Let $\Omega \subset \mathrm{R}^{2}$ and $\partial \Omega$ be a simple closed (smooth) curve. By parametrizing $\partial \Omega$ we may easily construct a family of periodic complex valued spline functions corresponding to a family of uniform subdivisions of $\partial \Omega$ of length $h$. If we take $S_{h}^{\prime} \subset H^{1}(\partial \Omega)$ for each $h$ then the condition (10.2) follows easily from the property $|\varphi|_{1} \leqq C h^{-1}|\varphi|_{0}$ which is easy to verify in view of the uniformity of the subdivisions. In addition, it is not difficult to see that the validity of (10.1) with $0 \leqq l \leqq k^{\prime}$ implies (10.1) for $-\frac{1}{2} \leqq l \leqq k^{\prime}$.

Now let $H=H^{1}(\Omega) \times H^{-1 / 2}(\partial \Omega)$ and let $U=\left\{u, u^{\prime}\right\} \in H$. (Here $u \in H^{1}(\Omega)$ and $u^{\prime} \in H^{-1 / 2}(\partial \Omega)$ are, of course, independent and $\|U\|_{I I}=\left(\|u\|_{1}^{2}+\left|u^{\prime}\right|_{-1 / 2}^{2}\right)^{1 / 2}$.) On $H \times H$ consider the sesquilinear form

$$
B(U, W)=B(u, w)-\left\langle u, w^{\prime}\right\rangle-\left\langle u^{\prime}, w\right\rangle,
$$

where $B$ is the form introduced in Section 4 . Now let $\left\{9 \pi_{h}\right\}_{0<h \leqq 1}$ be a family of subspaces of $H$ with $9 \pi_{h}=S_{\delta h} \times S_{h}^{\prime}$, $\delta$ a fixed small constant, $\left\{S_{h}\right\}_{0<h \leqq 1}$ of class $\delta_{1, r}$ and $\left\{S_{h}^{\prime}\right\}_{0<h \leqq 1}$ of class $S_{1 / 2, r-3 / 2}^{\prime}$ with $r \geqq 2$. Babuška [6] has proved the following inequality:

$$
\sup _{\Phi \in: M_{h} ;|\uparrow| \mid H \leqq 1}|O 3(U, \Phi)| \geqq C\|U\|_{H}
$$

for all $U \in \mathfrak{M}_{h}$, for a suitable positive constant $C$ independent of $h$ and $U$ provided that $\delta$ is sufficiently small. It is by means of this inequality that we may study a family of approximate solutions to (4.3) with $V=H_{0}^{1}(\Omega)$ (the Dirichlet problem). 
We first note that if $f \in \mathfrak{L}_{2}(\Omega)$ and $u$ is the solution of (4.3), then for $U=\{u, \partial u / \partial \nu\}$ and $\Phi=\left\{\varphi, \varphi^{\prime}\right\}$ arbitrary

$$
B(U, \Phi)=B(u, \varphi)-\langle\partial u / \partial \nu, \varphi\rangle=(f, \varphi) .
$$

By virtue of (10.3) there exists a unique $U_{h} \in 9 \pi_{h}$ satisfying

$$
B\left(U_{h}, \Phi\right)=(f, \varphi)
$$

for all $\Phi \in 9 \pi_{h}$. Now for $U_{h}=\left\{u_{h}, u_{h}^{\prime}\right\}$ define $T_{h} f=u_{h}$. Then, $T_{h}: \mathfrak{L}_{2}(\Omega) \rightarrow \mathfrak{L}_{2}(\Omega)$ and, since $S_{\delta h}$ is finite dimensional, $T_{h}$ is compact. As in Section 6 let $\mu(h)$ be a nonzero eigenvalue of $T_{h}$; i.e.,

$$
T_{h} w=\mu(h) w
$$

for some nonzero $w \in S_{h}$. Then with $\lambda(h)=1 / \mu(h)$ and $W=\left\{w, w^{\prime}\right\}$ for a certain $w^{\prime} \in S_{h}^{\prime}$, we have

$$
B(W, \Phi)=\lambda(h)(w, \varphi)
$$

for all $\Phi \in 9 \mathfrak{T}_{h}$. Hence, if we choose bases for $S_{\delta h}$ and $S_{h}^{\prime}$, then (10.7) reduces to a matrix eigenvalue problem with $\lambda(h)$ an eigenvalue. Conversely for $\lambda(h)$ satisfying (10.7) with $w$ a nonzero element of $\mathfrak{M}_{h}$ it follows easily that (10.6) holds with $\mu(h)=$ $1 / \lambda(h)$. Hence the nonzero eigenvalues $\mu(h)$ of $(10.6)$ may be determined from a finite-dimensional (matrix) eigenvalue problem.

Now to compare the eigenvalues of $T$ with those of $T_{h}$ we shall estimate the appropriate norms of $T-T_{h}$. Babuška [6] has already obtained some of the necessary estimates. Set $E=\left\{e, e^{\prime}\right\}$ where $e=u-u_{h}$ and $e^{\prime}=\partial u / \partial \nu-u_{h}^{\prime}$. He has shown that

$$
\|E\|_{H} \leqq C h^{t-1}\|f\|_{t-2}
$$

and

$$
\|e\|_{0} \leqq C h^{t}\|f\|_{t-2}
$$

for $2 \leqq t \leqq r$.

The method used for obtaining (10.9) also leads to the remaining desired estimates which, for completeness, we derive here.

Let $\psi \in C^{\infty}(\bar{\Omega})$ and set $V=\left\{T^{*} \psi, \partial T^{*} \psi / \partial \bar{\nu}\right\}$. We have that $(e, \psi)=B(E, V)$ and, using (10.4) and (10.5), $(e, \psi)=B(E, V-\Phi)$ for all $\Phi \in \mathfrak{T}_{h}$. Now by the trace inequality (cf. [26]), $|v|_{1 / 2} \leqq C\|v\|_{1}$ for $v \in H^{1}(\Omega)$, it follows immediately that $B$ is continuous on $H \times H$ and hence, since $\Phi$ is arbitrary,

$$
|(e, \psi)| \leqq C\|E\|_{H} \inf _{\phi \in \pi_{h}}\|V-\Phi\|_{H} .
$$

It is an immediate consequence of the properties of $9 \Upsilon_{h},(5.1)$ and $(10.1)$, that for $0 \leqq s \leqq r-2$,

$$
|(e, \psi)| \leqq C h^{s+1}\left(|| T^{*} \psi||_{s+2}+\left|\partial T^{*} \psi / \partial \bar{\nu}\right|_{s+1 / 2}\right)\|E\|_{H} .
$$

The trace inequality

$$
\left|\partial T^{*} / \partial \bar{\nu}\right|_{s+1 / 2} \leqq C \| T^{*} \psi||_{s+2}
$$

with $s \geqq 0$ is also well known (cf. [26]) so that 


$$
|(e, \psi)| \leqq\left. C h^{s+1}|| T^{*} \psi\left\|_{s+2}\right\| E\right|_{H} .
$$

We have then from (4.6) and (10.8) that

$$
|(e, \psi)| \leqq C h^{t+s}\|\psi\|_{s}\|f\|_{t-2} .
$$

Clearly, since $e=\left(T-T_{h}\right) f$, we have that

$$
\left\|T-T_{h}\right\|_{-s, t-2} \leqq C h^{t+s}
$$

for $0 \leqq s \leqq r-2$ and $2 \leqq t \leqq r$. Hence, in particular, setting $s=0$ and $t=2$, we see that

$$
\lim _{h \rightarrow 0}\left\|T-T_{h}\right\|_{0,0}=0
$$

so that Theorems 1-4 apply. Again the estimates (6.9) and (6.10) are valid.

11. $\mathscr{L}_{2}$-Projections. In each of the foregoing examples the approximate operators $T_{h}$ were chosen to be of the form $P_{h} T$ where $P_{h}$ is some projection onto $S_{h}$. The crucial point in all these Rayleigh-Ritz-Galerkin methods is that although the operator $T$ is usually not explicitly known, the operator $P_{h}$ is chosen in such a way that $P_{h} T$ can be explicitly evaluated. In case $T$ is an operator which can be evaluated, for example, if $T$ were given as an integral operator whose kernel is a known Green's function, then a possible choice of the operator $P_{h}$ is just the orthogonal projection in $\mathfrak{L}_{2}(\Omega)$ onto $S_{h}$.

As a final example we suppose that $T$ is an operator of the general type described in Section 3 and we take $T_{h}=\left.P_{h} T\right|_{\mathcal{L}_{2}(\Omega)}$, where $P_{h}$ is the orthogonal projection in $\mathfrak{L}_{2}(\Omega)$ onto $S_{h}$ with $\left\{S_{h}\right\}_{0<h \leqq 1}$ of class $S_{0, r}$. Assume that $r \geqq \epsilon$ where $\epsilon$ is as in Section 3 .

To obtain estimates for $T-T_{h}$ let $f \in H^{r-\epsilon}(\Omega)$ and $\psi \in C^{\infty}(\bar{\Omega})$. Then

$$
\begin{aligned}
\left|\left(\left(T-T_{h}\right) f, \psi\right)\right| & =\left|\left(\left(I-P_{h}\right) T f,\left(I-P_{h}\right) \psi\right)\right| \\
& \leqq \inf _{\chi_{1} \in S_{h}}\left\|T f-\chi_{1}\right\|_{0} \inf _{\chi_{2} \in S_{h}}\left\|\psi-\chi_{2}\right\|_{0} .
\end{aligned}
$$

It follows from (5.1) and (3.1) that

$$
\left|\left(\left(T-T_{h}\right) f, \psi\right)\right| \leqq C h^{s+t}\|\psi\|_{s}\|f\|_{t-\epsilon}
$$

for $0 \leqq s, t \leqq r$. Hence

$$
\left\|T-T_{h}\right\|_{-s, t-\epsilon} \leqq C h^{s+t}
$$

for $0 \leqq s, t \leqq r$ and in particular choosing $s=0$ and $t=\epsilon$ it follows that

$$
\lim _{h \rightarrow 0}\left\|T-T_{h}\right\|_{0,0}=0 \text {. }
$$

Thus Theorems 1-4 apply. Let $\mu$ be an eigenvalue of $T$ with algebraic multiplicity $m$ and $\hat{\mu}(h)=(1 / m) \sum_{i=1}^{m} \mu_{j}(h)$, where the $\mu_{j}(h)$ 's are the $m$ eigenvalues of $T_{h}$ which converge to $\mu$ as $h \rightarrow 0$. Then from Theorem 2 we find that, for $0<h \leqq h_{2}$,

$$
|\mu-\hat{\mu}(h)| \leqq C h^{2 r} .
$$

As before, for $\left\{u_{i}\right\}_{i=1}^{m}$ and $\left\{w_{i}\right\}_{i=1}^{m}$ the respective orthonormal bases for the corresponding invariant subspaces, we have from Theorem 1 that 


$$
\max _{1 \leqq i \leqq m}\left\|u_{i}-w_{j}\right\|_{0} \leqq C h^{r}
$$

\author{
Department of Mathematics \\ Cornell University \\ Ithaca, New York 14850
}

Department of Mathematics

University of Maryland

College Park, Maryland 20742

1. N. Aronszajn, "The Rayleigh-Ritz and A. Weinstein methods for approximation of eigenvalues. I, II," Proc. Nat. Acad. Sci. U.S.A., v. 34, 1948, pp. 474-480, 594-601. MR 10, 382.

2. N. AronszaJN, "Approximation methods for eigenvalues of completely continuous symmetric operators," Proc. Sympos. Spectral Theory and Differential Problems, Stillwater, Oklahoma, 1951, pp. 179-202. MR 13, 469.

3. N. Aronszajn \& A. Weinstein, "Existence, convergence, and equivalence in the unified theory of eigenvalues of plates and membranes," Proc. Nat. Acad. Sci. U.S.A., v. 27, 1941, pp. 188-191. MR 3, 44.

4. I. BABUŠKA, "Approximation by hill functions," Comment Math. Univ. Carolinae, v. 11, 1970, pp. 787-811.

5. I. BABUŠKA, Approximation by Hill Functions II, Technical Note BN-708, Institute for Fluid Dynamics and Applied Mathematics, University of Maryland, College Park, Md., 1971.

6. I. BabušKa, The Finite Element Method with Lagrangian Multipliers, Technical Note BN-724, Institute for Fluid Dynamics and Applied Mathematics, University of Maryland, College Park, Md., 1972.

7. N. W. BAZley, "Lower bounds for eigenvalues," J. Math. Mech., v. 10, 1961, pp. 289-308. MR 23 \#B1651.

8. N. W. BAZley \& D. W. Fox, "Truncations in the method of intermediate problems for lower bounds to eigenvalues," J. Res. Nat. Bur. Standards Sect. B, v. 65B, 1961, pp. 105111. MR 26 \#464.

9. N. W. BAzley \& D. W. Fox, "A procedure for estimating eigenvalues," J. Mathematical Phys., v. 3, 1962, pp. 469-471. MR 26 \#1998.

10. Ju. M. BerEZANSKII, Expansion in Eigenfunctions of Self Adjoint Operators, Naukova Dumka, Kiev, 1965; English transl., Transl. Math. Monographs, vol. 17, Amer. Math. Soc., Providence, R. I., 1968. MR 36 \#5768; 36 \#5769.

11. G. BirkhofF, C. DE Boor, B. Swartz \& B. Wendroff, "Rayleigh-Ritz approximation by piecewise cubic polynomials," SIAM J. Numer. Anal., v. 3, 1966, pp. 188-203. MR 34 \#3773.

12. J. H. Bramble, T. Dupont \& V. Thomée, "Projection methods for Dirichlet's problem in approximating polygonal domains with boundary-value corrections," Math Comp., v. 26,1972 , pp. $869-879$.

13. J. H. Bramble \& S. R. Hilbert, "Estimation of linear functionals on Sobolev spaces with application to Fourier transforms and spline interpolation," SIAM J. Numer. Anal., v. 7, 1970, pp. 112-124. MR 41 \#7819.

14. J. H. Bramble \& S. R. Hilbert, "Bounds for a class of linear functionals with application to Hermite interpolation," Numer. Math., v. 16, 1970/71, pp. 362-369. MR 44 \#704.

15. J. H. Bramble \& A. H. Schatz, "Rayleigh-Ritz-Galerkin methods for Dirichlet's problem using subspaces without boundary conditions," Comm. Pure Appl. Math., v. 23, 1970, pp. 653-675. MR 42 \#2690.

16. J. H. Bramble \& A. H. Schatz, "Least squares methods for $2 m$ th order elliptic boundary-value problems," Math. Comp., v. 25, 1971, pp. 1-32.

17. J. H. Bramble \& M. Zlámal, "Triangular elements in the finite element method," Math. Comp., v. 24, 1970, pp. 809-820. MR43 \#8250.

18. P. G. Ciarlet \& P.-A. Raviart, "Interpolation theory over curved elements, with application to finite element methods," Computer Methods in Appl. Mech. and Engineering, v. 1, 1972, pp. 217-249.

19. P. G. Ciarlet, M. H. Schultz \& R. S. Varga, "Numerical methods of high-order accuracy for nonlinear boundary value problems. I'I. Eigenvalue problems," Numer. Math., v. 12, 1968, pp. 120-133. MR 38 \#1838.

20. N. Dunford \& J. T. SchwarTZ, Linear Operators. II: Spectral Theory. Selfadjoint Operators in Hilbert Space, Interscience, New York, 1963. MR 32 \#181. 
21. G. Fichera, "Approximation and estimates for eigenvalues," in Numerical Solution of Partial Differential Equations (Proc. Sympos. Univ. Maryland, 1965) (Edited by J. H. Bramble), Academic Press, New York, 1966, pp. 317-352. MR 36 \#733.

22. G. FICHERA, "Further developments in the approximation theory of eigenvalues," in Numerical Solution of Partial Differential Equations, II (SYNSPADE 1970) (Proc. Sympos. Univ. of Maryland, 1970) (Edited by B. Hubbard), Academic Press, New York. 1971, pp. 243-252. MR 43 \#2841.

23. F. DI Guglielmo, "Construction d'approximations des espaces de Sobolev sur des réseaux en simplexes," Calcolo, v. 6, 1969, pp. 279-331.

24. S. Hilbert, Numerical Methods for Elliptic Boundary Problems, Thesis, University of Maryland, College Park, Md., 1969.

25. T. Kato, Perturbation Theory for Linear Operators, Die Grundlehren der math. Wissenschaften, Band 132, Springer-Verlag, New York, 1966. MR 34 \#3324.

26. J. L. Lions \& E. Magenes, Problèmes aux Limites non Homogènes et Applications.

Vol. I, Travaux et Recherches Mathématiques, no. 17, Dunod, Paris, 1968. MR 40 \#512.

27. I. MAREK, Approximation of the Principal Eigenelements in K-Positive Non SelfAdjoint Eigenvalue Problems, MRC Technical Summary Report \#1094, University of Wisconsin, Madison, Wis., 1971.

28. J. NiTSCHE, "Über ein Variationsprinzip zur Lösung von Dirichlet-Problemen bei Verwendung von Teilräumen, die keinen Randbedingungen unterworfen sind," $A b h$. Math. Sem. Univ. Hamburg, v. 36, 1970/71.

29. J. NiTSCHE, "A projection method for Dirichlet-problems using subspaces with nearly zero boundary conditions." (Preprint.)

30. J. E. OsBoRN, "Approximation of the eigenvalues of non self-adjoint operators," $J$. Math. and Phys., v. 45, 1966, pp. 391-401. MR $34 \# 8189$.

31. J. E. OsboRn, "Approximation of the eigenvalues of a class of unbounded, non selfadjoint operators," SIAM J. Numer. Anal., v. 4, 1967, pp. 45-54. MR. 35 \#458.

32. J. E. OsBorN, "A method for approximating the eigenvalues of non self-adjoint ordinary differential operators," Mem. Accad. Sci. Torino Cl. Sci. Fis. Mat. Natur. (4), no. 14. pp. $1-56$.

33. J. G. Pierce \& R. S. VARGa, "Higher order convergence results for the Rayleigh-Ritz method applied to eigenvalue problems: I. Estimates relating Raleigh-Ritz and Galerkin approximations to eigenfunctions," SIAM J. Numer. Anal., v. 9, 1972, pp. 137-151.

34. J. G. PIERCE \& R. S. VARGA, "Higher order convergence results for the Rayleigh-Ritz method applied to eigenvalue problems: II. Improved error bounds for eigenfunctions", Numer. Math., v. 19, 1972, pp. 155-169.

35. M. Schechter, "On $L^{p}$ estimates and regularity I," Amer. J. Math., v. 85, 1963, pp. 1-13. MR 32 \#6051.

36. M. Schechter, "On $L^{p}$ estimates and regularity II," Math. Scand., v. 13, 1963, pp. 47-69. MR $32 \# 6052$.

37. I. J. Schoenberg, "Contributions to the problem of approximation of equidistant data by analytic functions," Quart. Appl. Math., v. 4, 1946, part A, pp. 45-99, part B, pp. 112-141. MR 7, 487; 8, 55 .

38. M. H. Schultz, "Rayleigh-Ritz-Galerkin methods for multi-dimensional problems," SIAM J. Numer. Anal., v. 6, 1969, pp. 523-538. MR 41 \#7859.

39. M. H. Schultz, "Multivariate spline functions and elliptic problems," in Approximation with Special Emphasis on Spline Functions (Edited by I. J. Schoenberg), Academic Press, New York, 1969, pp. 279-347. MR 41 \#2210.

40. M. H. Schultz, " $L^{2}$ error bounds for the Rayleigh-Ritz-Galerkin method," SIAM $J$. Numer. Anal., v. 8, 1971, pp. 737-748.

41. W. STENGER, "On the variational principles for eigenvalues for a class of unbounded operators," J. Math. Mech., v. 17, 1967/68, pp. 641-648. MR 37 \#3384.

42. G. M. VAYNIKKO, "Asymptotic evaluation of the error of projection methods for the eigenvalue problem," Ž. Vyčisl. Mat. i Mat. Fiz., v. 4, 1964, pp. 405-425. USSR Comput. Math. and Math. Phys., v. 4, 1964, pp. 9-36.

43. G. M. VAINIKKo, "On the rate of convergence of certain approximation methods of Galerkin type in an eigenvalue problem," Izv. Vyš̌s. Učebn. Zaved. Matematika, 1966, no. 2(51), pp. 37-45; English transl., Amer. Math. Soc. Transl. (2), v. 86, 1970, pp. 249-259. MR 33 \#6824; 41 \#1462.

44. G. M. VainikKo, "On the speed of convergence of approximate methods in the eigenvalue problem," Ž. Vyčisl. Mat. i Mat. Fiz., v. 7, 1967, pp. 977-987. USSR Comput. Math. and Math. Phys., v. 7, 1967, pp. 18-32.

45. H. F. WeInbERGER, "Error estimation in the Weinstein method for eigenvalues," Proc. Amer. Math. Soc., v. 3, 1952, pp. 643-646. MR 14, 290.

46. H. F. Weinberger, $A$ Theory of Lower Bounds for Eigenvalues, Technical Note BN183, Institute for Fluid Dynamics and Applied Mathematics, University of Maryland, College Park, Md., 1959.

47. A. Weinstein, "Sur la stabilité des plaques encastrées," C. R. Acad. Sci. Paris, v. 200, 1935, pp. $107-109$. 
48. A. WeINSTEIN, "Étude des spectres des équations aux dérivées partielles de la théorie des plaques élastiques," Mem. Sci. Math., v. 88, 1937.

49. A. WEINSTEIN, "Bounds for eigenvalues and the method of intermediate problems," Proc. Internat. Conf. Partial Differential Equations and Continuum Mechanics, Univ. of Wisconsin Press, Madison, Wis., 1961, pp. 39-53. MR 23 \# A3365.

50. A. WEINSTEIN, "A necessary and sufficient condition in the maximum-minimum theory of eigenvalues," Studies in Math. Anal. and Related Topics, Stanford Univ. Press, Stanford, Calif., 1962, pp. 429-434. MR 26 \#142.

51. A. WEINSTEIN, "Intermediate problems and the maximum-minimum theory of eigenvalues," J. Math. Mech., v. 12, 1963, pp. 235-246, MR 27 \# 5025.

52. A. Weinstein, "An invariant formulation of the new maximum-minimum theory of eigenvalues," J. Math. Mech., v. 16, 1966, pp. 213-218. MR 35 \#3475.

53. O. C. ZIENKIEWICZ, The Finite Element Method in Structural and Continuum Mechanics, McGraw-Hill, New York, 1967.

54. M. ZláMal, "On the finite element method," Numer. Math., v. 12, 1968, pp. 394-409. MR 39 \# 5074.

55. M. Zlámal, "Curved elements in the finite element method.” (Preprint.) 